\title{
Agôn
}

Revue des arts de la scène

$8 \mid 2019$

Matières

\section{Marionnette infigurée et persistance de la matière}

Julie Postel

\section{(2) OpenEdition}

\section{Journals}

Édition électronique

URL : http://journals.openedition.org/agon/6114

DOI : 10.4000/agon. 6114

ISSN : 1961-8581

Éditeur

Association Agôn

Référence électronique

Julie Postel, « Marionnette infigurée et persistance de la matière », Agôn [En ligne], 8 | 2019, mis en ligne le 15 décembre 2019, consulté le 10 décembre 2020. URL : http://journals.openedition.org/agon/ 6114 ; DOI : https://doi.org/10.4000/agon.6114

Ce document a été généré automatiquement le 10 décembre 2020.

Association Agôn et les auteurs des articles 


\title{
Marionnette infigurée et persistance de la matière
}

\author{
Julie Postel
}

Pour la création de la " déambulation théâtralisée ${ }^{1}$ ", Des hurlements montaient le long des saules pleureurs ${ }^{2}$ (2013), François Lazaro, directeur artistique du Clastic Théâtre, installe dans le vaste espace d'une forge ardennaise les pantins avachis et immobiles sculptés par Francis Marshall [Figure 1]. Ces objets inarticulés s'ancrent de tout leur poids dans un espace chargé d'histoire, que les spectateur.trice's traversent à la tombée de la nuit. N'étant pas manipulés par les interprètes, les pantins anthropomorphes entrent dans un dialogue virtuel avec les machines de forges gigantesques et immobiles, sans que ni les uns ni les autres ne soient jamais érigés de façon univoque en sujets du drame, jamais tout à fait animés. Des présences fragiles naissent à l'entour d'eux, par la résonance des voix, la circulation des lumières, et le mouvement des interprètes à travers les lieux. 
Figure 1

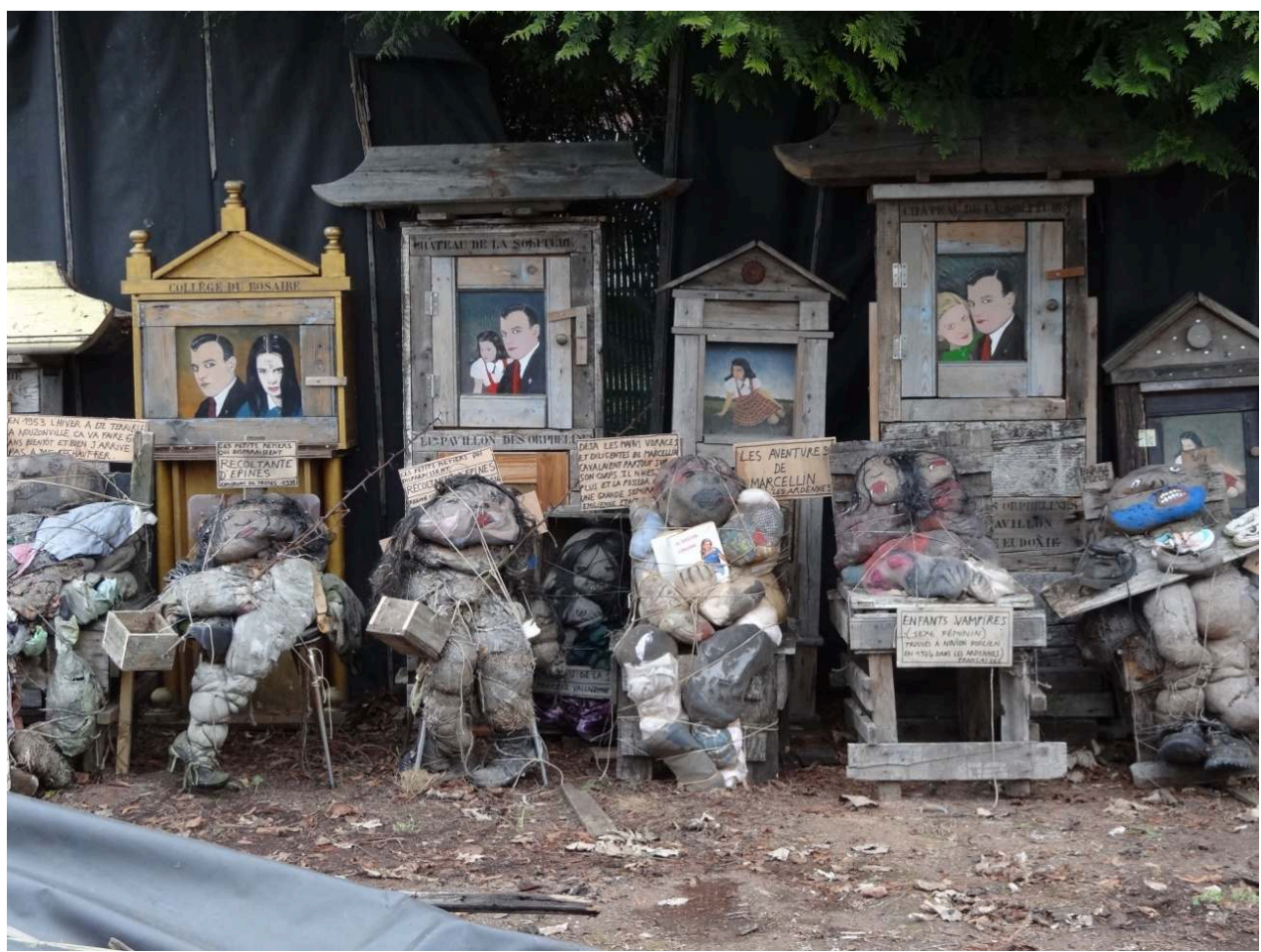

Sculptures de Francis Marshall dans son jardin, 2013 (Photo : Clastic Théâtre)

2 Dans les créations de Gisèle Vienne, telles I apologize (2004), la difficile identification d'un corps fixe pour la figure dramatique passe également par la mise en scène de l'inertie des objets [Figure 2]. 
Figure 2

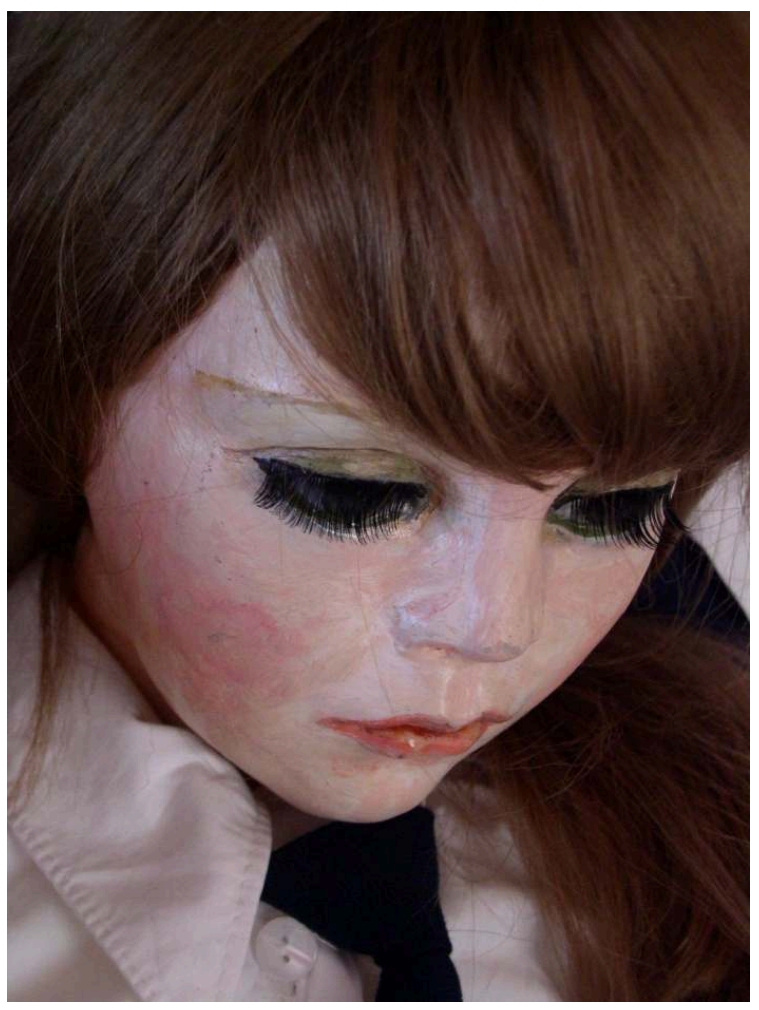

I apologize, Gisèle Vienne, 2004 (Photo : Gisèle Vienne @ DACM)

3 La présence circule à travers une multiplicité de corps, humains et non-humains, que le public reconnaît alternativement comme des corps habités ou comme de simples images de corps, vidées de toute subjectivité. Cette circulation produit en creux l'effet d'une désanimation inquiétante des corps-objets, dès lors qu'ils se trouvent abandonnés à leur matérialité inerte de poupées.

4 La compagnie Les Rémouleurs élabore également dans Rêves et motifs (2017) une figure aux multiples formes et visages, étendue à travers les éléments du dispositif : corps, objets, matières brutes, dessins [Figure 3]. La compagnie travaille en particulier sur l'image projetée comme lieu de l'animation. Au centre du dispositif bifrontal de Rêves et motifs se trouve un " miroir liquide », qui est un écran de savon³. 
Figure 3

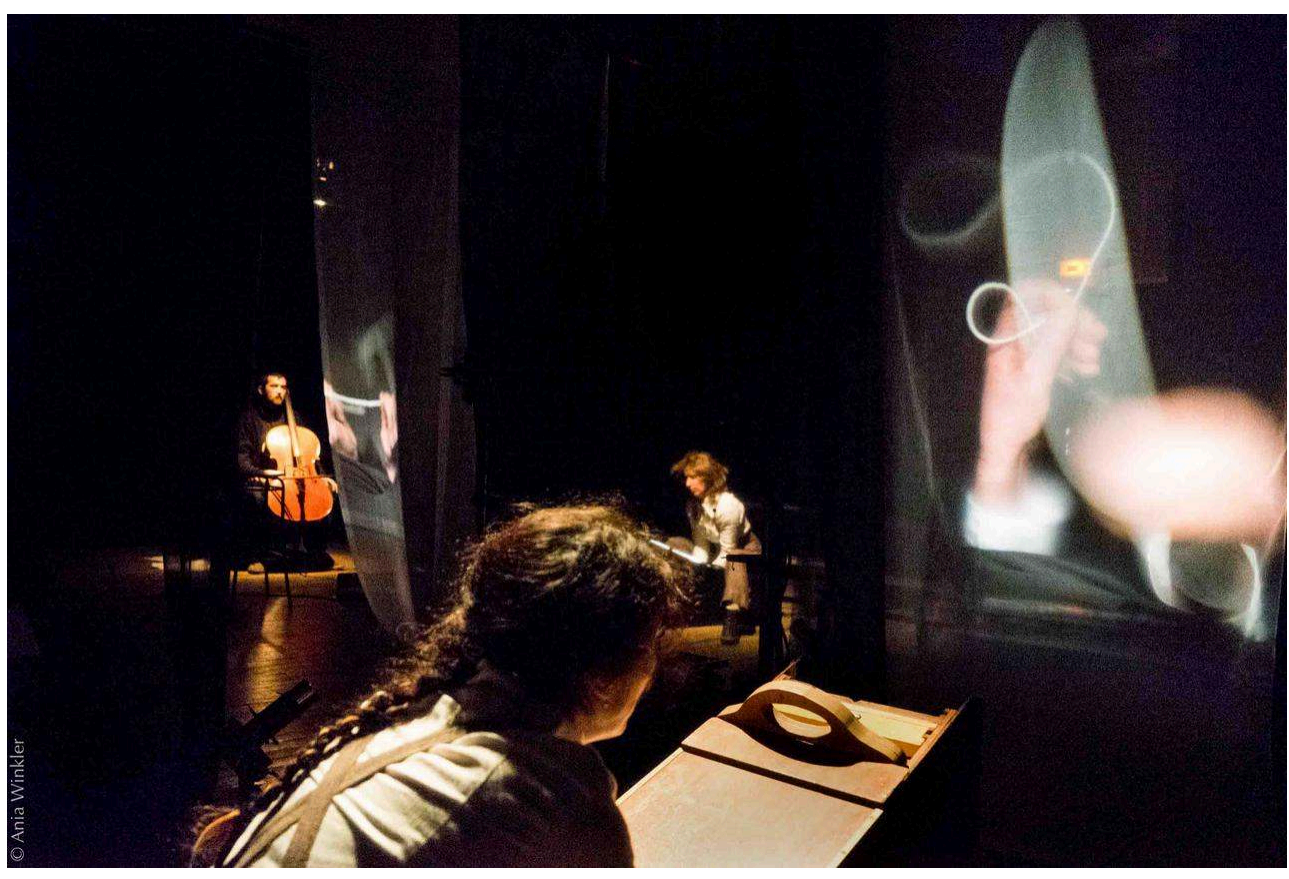

Rêves et motifs, Les Rémouleurs, 2017 (Photo : Ania Winkler)

5 Le film de savon confère un caractère fragile et abstrait à la forme qui s'y dessine. Apparaissent des formes fugaces, des reflets éphémères d'une figure qui semble habiter l'espace du plateau mais n'y prendre jamais corps de façon définitive. Ainsi éclatée dans l'espace de jeu, la pensée du mathématicien Alexandre Grothendieck - dont les écrits constituent le fil dramaturgique du projet - en devient omniprésente, bien qu'elle n'apparaisse à la surface du visible que de façon discontinue et sous forme d'images évanescentes.

6 L'artiste flamand Benjamin Verdonck crée quant à lui des castelets mobiles ouvrant sur des présences infigurées. Dans sa création Chansonnette pour Gigi (2018) comme dans son précédent Notallwhowanderarelost (2014) [Figure ], des constructions géométriques très épurées, mises en mouvement par des ficelles, ne se constituent pas en corps de la figure marionnettique mais plutôt en indices minimaux de l'élaboration de présences invisibles. 
Figure 4

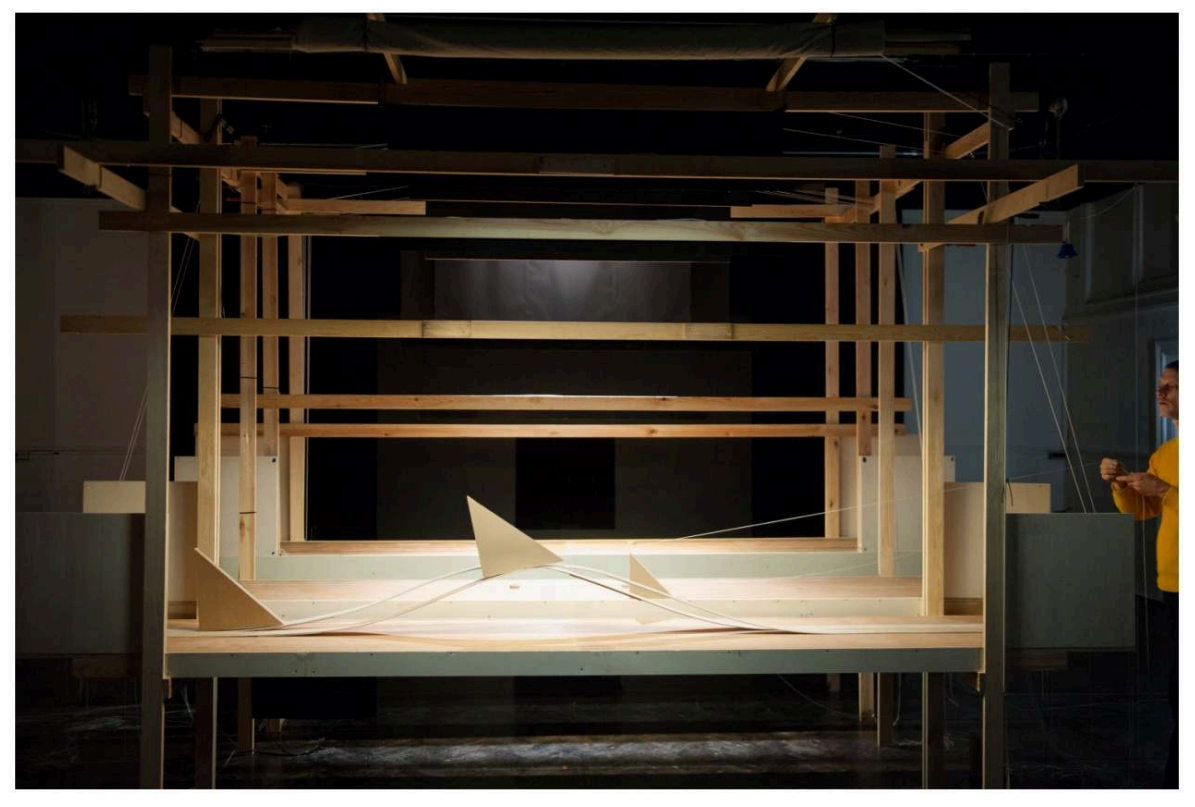

Notallwhowanderarelost, Benjamin Verdonck, 2014 (Photo : Kurt van der Elst)

7 Bien qu'elles n'aient pas de corps, les figures agissant dans Chansonnette pour Gigi [Figure 5] se manifestent entre les pans de murs du castelet animé. Elles existent grâce aux jeux de lumière, à la musique, au texte dit.

\section{Figure 5}

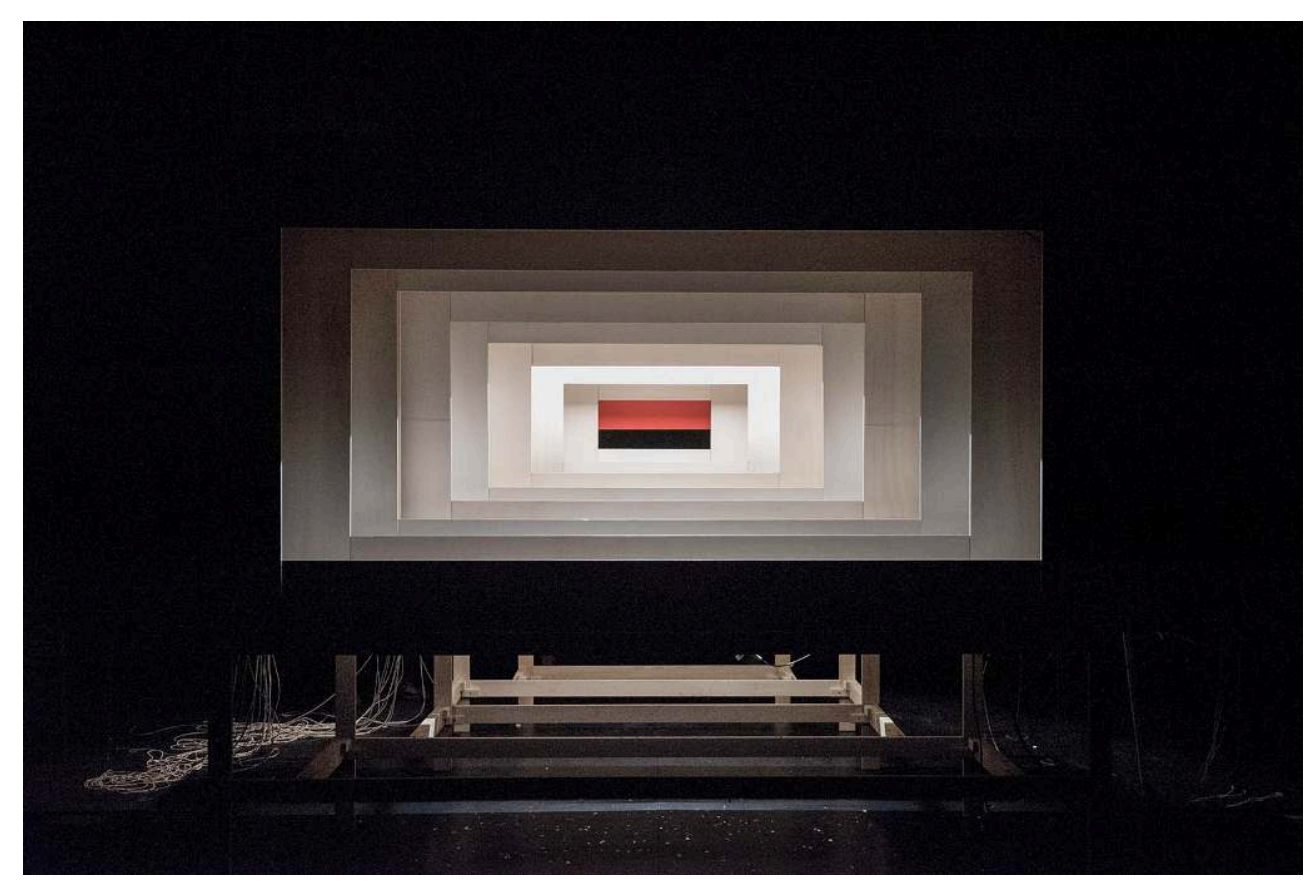

Chansonnette pour Gigi, Benjamin Verdonck, 2018 (Photo : Kurt van der Elst) 
Si nous avons choisi d'ouvrir notre article par cette série d'instantanés tirés de créations récentes, aux esthétiques et dramaturgies manifestement hétérogènes, c'est que toutes ont au moins une chose en commun : elles déstabilisent une définition des arts de la marionnette qui serait centrée sur l'objet manipulé. Certes, ces artistes détournent le principe d'incarnation et mettent en œuvre des présences sans visages ni corps humains. Certes, un important travail plastique préside à leurs créations et les contraintes matérielles que posent ces dispositifs en termes de montage, de stockage, de coût et temps de production, attirent notre attention sur une filiation de ces expériences avec les arts de la marionnette. Pourtant, les spectateur.trice-s assistent dans ces œuvres à une disparition - parfois ponctuelle ou seulement partielle - du corps de « la marionnette » : les objets visibles et mis en mouvement ne sont pas érigés en corps de la figure dramatique. Ils ne se constituent pas en sièges univoques d'une présence.

Le préconçu mis à mal par ces expériences est donc celui d'une marionnette pensée comme "objet-marionnette", c'est-à-dire réductible à un objet fixe, unique et identifiable qui, par l'acte de manipulation, serait érigé en corps d'un sujet fictif ${ }^{4}$. En d'autres termes, les créations contemporaines qui retiennent notre attention défont une conception iconique et sémiologique de la marionnette, qui associerait de façon univoque un corps matériel à une instance dramatique idéelle ${ }^{5}$. Une telle conception ne permet pas de saisir l'économie des figures mobiles, fugaces et incertaines, existant par le mouvement de formes instables ou par le dialogue immobile des matériaux. La figure marionnettique existe et agit alors davantage entre les corps.

Ce que nous entendons comme défection de l'objet-marionnette n'est pourtant pas synonyme de dématérialisation de l'ensemble du dispositif marionnettique. Il s'opère plus précisément une dissociation entre le geste plastique et le geste d'animation : les lieux de l'animation ne sont plus ceux de la matière, bien que la matière persiste.

La question à partir de laquelle nous souhaitons donc interroger ces créations contemporaines est celle du nécessaire passage par la matière dans la mise en œuvre de présences marionnettiques. La conception hégélienne des rapports de l'art à la matière, rapportée par Florence de Mèredieu dans son Histoire matérielle et immatérielle de l'art moderne, éclaire une hypothèse intéressante qui serait celle de la tension vers la matérialisation « la plus minime» :

L'art ne peut se passer de la matière, d'une incarnation hic et nunc, dans les dimensions plurielles de l'espace et du temps. Cette incarnation fut-elle [...] la plus minime et la plus "immatérielle" possible ! 6

9 L'idée d'un passage, nécessaire et minimal, par la matière éclaire le devenir de la figure marionnettique contemporaine: instance dramatique en tension vers l'infiguré et pourtant irréductiblement liée à l'organisation matérielle de la représentation et au système des objets scéniques.

Notre analyse éclairera dans un premier temps le déplacement de la fonction figurale, de l'objet vers « l'entre » des corps et des matières, la défiguration passant par la mise en scène du délitement des formes. Le devenir infiguré de la marionnette procède de la mise en tension des espaces au creux du visible. Alors la disparition de «l'objetmarionnette ", en tant que fonction figurale attribuée à un objet fixe, nous permettra d'envisager la figure marionnettique comme ondulatoire et immatérielle, existant par l'orchestration des multiples médiums ${ }^{7}$ visuels et sonores.

Puis l'observation attentive de ce que fait la matière dans ces processus marionnettiques nous révèlera dans un second temps un déplacement de son fonctionnement 
dramaturgique: les modes de passage par la matière témoignent non plus d'une démarche de transfiguration mais davantage d'une mise en drame de la capacité de résistance, de persistance de la matière. Elle agit non plus comme médium « transparent » mais comme reste en suspens, « opacifié " ${ }^{8}$. Le drame tient à son être-là propre. Il est lié au caractère irrémédiable et concret de son existence physique.

\section{Vers des présences marionnettiques infigurées ou la marionnette impondérable}

10 Les créations du Clastic Théâtre, de Gisèle Vienne, des Rémouleurs et de Benjamin Verdonck mettent en échec la reconnaissance d'un objet-marionnette dans la mesure, d'abord, où les choix plastiques réalisés par les artistes tendent à l'effacement des frontières matérielles de l'instance animée. Le travail avec des matériaux en voie de décomposition, la multiplication des objets, l'abstraction des formes, défont l'unité et l'unicité de l'objet-marionnette. Un principe traversant l'ensemble des créations qui retiennent notre attention est donc celui de la mise en drame d'une tension vers l'informe, que nous nommerons défiguration marionnettique, et qui tend à l'infiguration, l'effacement des visages et des corps, l'effacement des figures.

\section{Figures en décomposition : dégradation matérielle et abstraction des formes}

11 La création des Hurlements du Clastic Théâtre est née du double désir de François Lazaro de rendre hommage aux œuvres textuelles, picturales et sculpturales de Francis Marshall ${ }^{9}$ et de s'inscrire dans un territoire marqué par l'histoire ouvrière. Au moment des représentations, en 2013, la forge FECR Production, choisie comme lieu de la création, est la dernière forge encore en activité dans les Ardennes. Cette création prend ainsi la forme d'un dialogue entre des sculptures faites de matériaux usagés et récupérés, l'écriture dramatique de François Lazaro et un contexte local marqué par la crise de la désindustrialisation ${ }^{10}$.

Les pantins de Francis Marshall se présentent comme des corps-rebuts. Le sculpteur travaille avec des matériaux marqués par leur usure et leur fragilité, collants bourrés de laines, de paille et de chiffons, sculptures faites de bois flotté, de tissus vieillis et raccommodés [Figure 6]. L'artiste encourage d'ailleurs la décomposition de ses œuvres en les conservant dans son jardin, à l'air libre. 
Figure 6

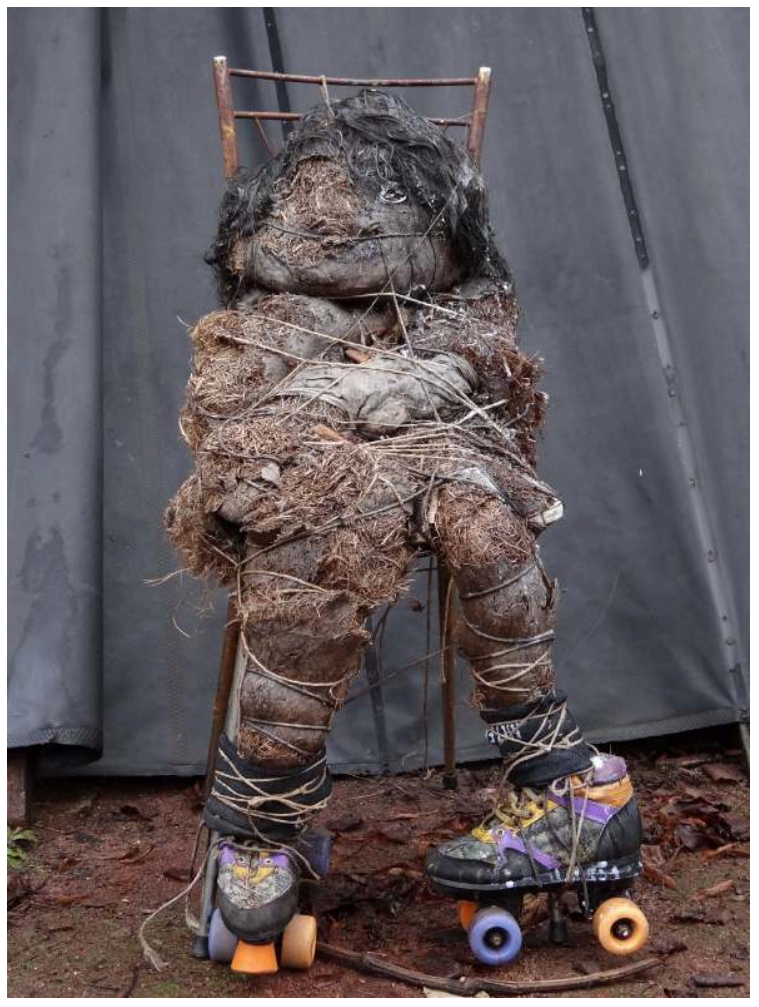

Josy, Francis Marshall, 2013 (Photo : Clastic Théâtre)

12 Ces corps-objets, bien qu'inertes, portent donc la trace matérielle d'une évolution en cours. La matière en décomposition indique une progression de ces corps vers une fin inéluctable. Leurs coutures ont craqué, leurs bouches peintes s'effacent. Ces sculptures anthropomorphes aux visages extrêmement ronds et aux membres potelés ont par ailleurs l'air gonflées jusqu'au trop-plein. Le plasticien qualifie lui-même ses pantins de " bourrages ${ }^{11}$ ». Çà et là, une jambe ou un visage se trouvent déjà éclatés, défigurés par des entrailles de tissus et de crins qui se répandent en-dehors du corps. En outre, Francis Marshall n'efface pas les traces de son travail d'assemblage. Ficelles et nœuds laissent visibles ses rafistolages et lacèrent les corps [Figure 7]. Ils déposent sur ces objets le sceau d'une cohésion matérielle à la fois laborieuse et fragile. La visibilité du travail d'assemblage souligne l'hétérogénéité des objets et dramatise la potentielle rupture de leur organicité. 
Figure 7

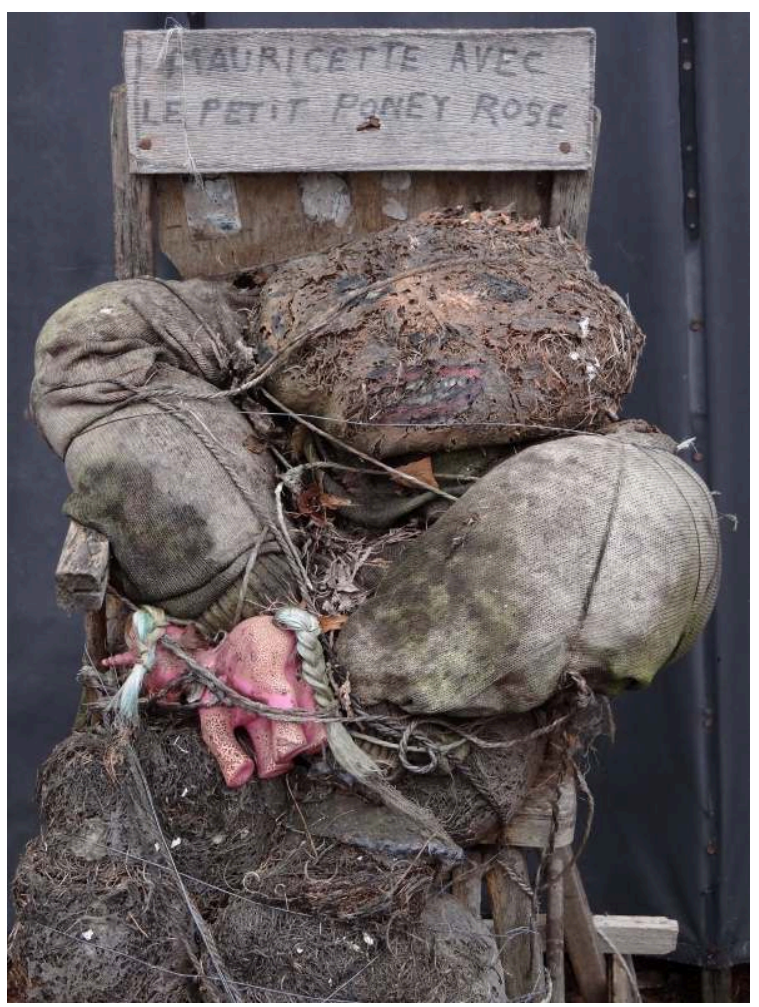

Mauricette avec le petit poney rose, Francis Marshall, 2013 (Photo : Clastic Théâtre)

Dans les Hurlements, la mise à mal du principe d'objet-marionnette tient donc d'abord à la mise en scène d'une défiguration en cours. Ce délitement tient à la projection d'une mémoire sur la matière, et à la perception d'une explosion qui menace. La mise en scène du Clastic Théâtre rend compte de ce drame plastique enclos par la confrontation de ces objets aux machines de forge abandonnées par la main ouvrière. L'installation des pantins de Francis Marshall dans ces lieux, que l'on traverse en soirée, crée d'emblée un dialogue sourd et immobile entre des corps en voie de décomposition et les grandes machines de fer, à l'arrêt, elles aussi menacées de disparition et d'immobilité. Ainsi le lent processus de défiguration des sculptures de Marshall rend tangible dans la mise en scène de François Lazaro une menace de disparition qui pèse sur la présence humaine dans le lieu symbolique de la forge.

Alors que le Clastic Théâtre met en scène l'effacement en cours de la forme humaine par le biais de la matière en décomposition, le visage humain est autrement effacé des créations de Benjamin Verdonck, qui mettent en jeu de simples formes géométriques. L'abstraction des formes y défait le principe d'objet-marionnette, dans la mesure où l'espace vide entre les objets peut être autant investi de présence que les formes matérialisées elles-mêmes. La dynamique dramatique tient davantage à la relation des couleurs ainsi qu'au mouvement des formes. Ainsi, l'artiste s'inscrit dans la lignée d'Oskar Schlemmer et de son Ballet triadique (1922) ${ }^{12}$ lorsqu'il met en scène de simples triangles traversant un cadre rectangulaire [Figure 8]. 


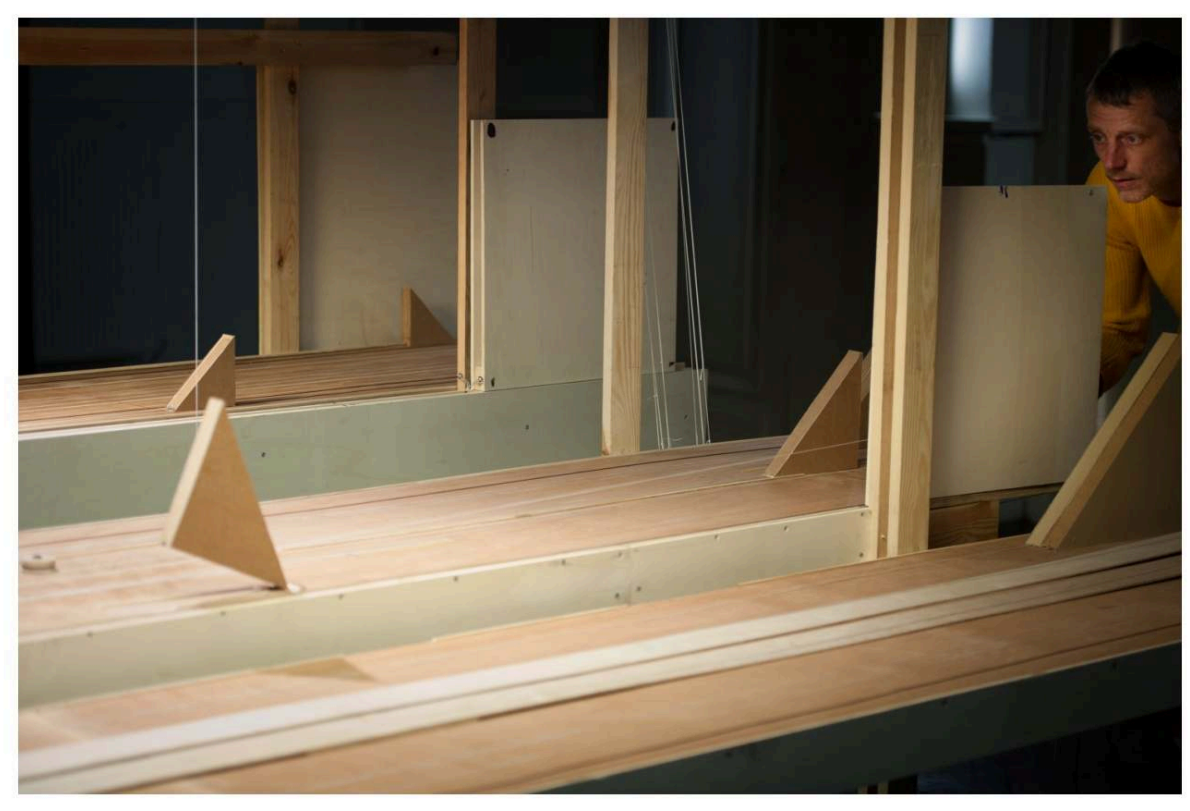

Notallwhowanderarelost, Benjamin Verdonck, 2014 (Photo : Kurt van der Elst) délitement matériel de l'objet-marionnette. À défaut d'être érigées en corps d'un sujet dramatique (comme le seraient des objets-marionnettes), les formes visibles constituent des indices minimaux d'une action dramatique. À propos des triangles manipulés par des ficelles dans Notallwhowanderarelost, et dont seule la taille varie, l'artiste évoque " une version schématique de l'acteur quand il est sur scène ${ }^{13}$ ». Le triangle ouvre selon lui sur une multitude de figures possibles mais l'intéresse surtout pour la dynamique, le potentiel de transformation et le mouvement que cette forme permet:

J'aimais cette forme parce que c'est la forme la plus simple. Il suffit de trois lignes pour faire cette forme. On peut aussi faire un rond mais [...] le rond, on ne peut pas le faire changer alors que le triangle, on peut jouer sur une ligne ou sur l'autre. Et puis, il me semblait qu'on pouvait y voir des bateaux, des acteurs ou encore des automobiles. C'était une forme qui ouvrait beaucoup de possibilités. [...] Pour moi, cette forme était une marque, un indice que quelque chose allait d'un endroit à un autre. La forme en elle-même était moins importante que le mouvement qu'elle rendait possible. ${ }^{14}$

La mise en jeu d'objets plans et géométriques, tous de forme identique, suppose une réduction de l'objet au statut d'indice plastique d'un drame cinétique qui le déborde. La qualité physique et le potentiel chorégraphique des formes mues résulte d'une recherche plastique du plus grand effacement possible des corps et des figures. Benjamin Verdonck parle d'ailleurs à propos des couleurs qu'il choisit pour ces formes - gris, pastels, jaune pâle - de «non-couleurs »" Il réduit matériellement ses objets à des marqueurs de présences mobiles, marqueurs minimaux mais suffisants. Ainsi ses castelets animés défont l'assignation - définitoire de l'objet-marionnette d'un objet fixe à la fonction d'incorporation d'un sujet déterminé. 
Enfin, un pas de plus est franchi dans la défiguration de l'objet-marionnette dès lors que des artistes choisissent de mettre en scène des matériaux bruts, c'est-à-dire n'ayant fait l'objet d'aucun travail de mise en forme préalable à leur mise en jeu au plateau. Les Rémouleurs travaillent par exemple dans Rêves et motifs avec du papier froissé qui peut à loisir se déployer dans l'espace ou être réduit à une boule chiffonnée. Certes, une recherche plastique a été menée par eux pour obtenir de ce papier une plasticité et une transparence singulières - le papier utilisé a été oint à l'huile de lin et longtemps mis à sécher - mais la matière mise en scène adopte des formes éphémères. La mise en scène du geste de sculpture met en tension avec la forme visible toutes les formes possibles, qui pourraient exister à travers cette matière. Bien qu'elles n'existent que virtuellement, ces formes agissent en puissance dans l'imaginaire spectatoriel et débordent la simple feuille de papier. Elles dépassent la fonction figurative que l'on aurait conférée à un objet-marionnette.

Dans le paysage plus large des arts de la marionnette, certainee's artistes concentrent même leurs recherches sur la mise en scène de matériaux fluides, échappant irréductiblement à la forme. Nous pouvons à cet égard citer les travaux de Pierre Meunier de la compagnie La Belle Meunière (La Vase, 2017), ceux d'Arnaud Louski-Pane, qui travaille avec des vapeurs, des souffles d'air, des mousses et des fumées (To fall is to understand the universe, 2013; Les Hautes Herbes, 2017), d'Élise Vigneron, qui a mis en scène à la suite d'Émilie Valantin ${ }^{16}$ des marionnettes de glace (Anywhere, 2016), ou encore de Phia Ménard et son cycle de recherche sur « l'Injonglabilité Complémentaire des Éléments (I.C.E.) $»^{17}$. Leurs recherches visent à saisir le potentiel dramatique propre à chaque matière et ses qualités physiques singulières, mais surtout, l'impossibilité de toute forme recentre le drame sur la relation entre les corps, les matières et sur la dynamique (dramatique) des fluides.

Les figures que ces artistes érigent en scène possèdent une présence et une force d'action dramatique quoiqu'elles échappent à toute fixation dans le visible. Ces démarches appellent une redéfinition du geste marionnettique dans la mesure où elles réinventent l'articulation entre geste plastique et geste dramatique. Le geste d'animation - celui qui crée des présences en-dehors des corps vivants - ne succède pas au geste sculptural. Soit il se confond avec lui, soit il naît de l'échec d'un geste plastique qui pourtant fait exister une relation. Alors la figure marionnettique existe sans visage, avant le visage : elle se trouve défigurée et en tension vers l'infiguré tant qu'elle se refuse à la fixation dans une forme modelée, discernable.

\section{Apparitions : des figures à la lisière du visible et de l'invisible}

Ce qui agit à travers la manipulation de matériaux fluides ou de matériaux bruts sculptés à vue est la mise en tension du vide et du plein, de la forme visible et de la forme potentielle, celle existant avant tout dans l'imaginaire spectatoriel par projection et par anticipation. De même, la disparition en cours des objets de Francis Marshall, mise en scène par le Clastic Théâtre, comme les formes épurées de Benjamin Verdonck, placent au cœur des dramaturgies cette tension entre le visible et l'invisible, par laquelle la figure marionnettique acquiert une présence au-delà de la matière. L'injonction de Valère Novarina à " creuser la matière profonde jusqu'à monter au trou aérien ${ }^{18}$ " fait écho à ces figures impondérables. Or, leur circulation sur le mode ondulatoire est souvent rendue perceptible par la manipulation du son et de la lumière. Grâce à elle, la figure agit au creux des formes, à travers l'ensemble de l'espace 
dramatique.

Outre le traitement plastique des médiums ondulatoires que sont le son et la lumière, la dématérialisation peut être mise en scène comme procès par l'écriture du devenirimage de l'objet animé. Olivier Vallet, co-directeur artistique de la compagnie Les Rémouleurs, mène des recherches sur l'histoire des techniques de projection d'images. Il réhabilite des techniques ayant été délaissées et peu exploitées pour réinventer, en collaboration avec des scientifiques, des machines productrices d'images. La portée dramatique de ses inventions tient à son travail sur l'épaisseur matérielle des images produites. En effet, Olivier Vallet travaille à la fois sur la source de l'image (la machine de projection elle-même), sur le support de projection et sur le dispositif qui les articule. Son travail implique une véritable conception plastique et spatialisée des images. Les créations des Rémouleurs, telles Rêves et motifs, mettent ainsi en scène tout le processus de figuration comme un cheminement dramatique réalisé à vue, et qui vise l'apparition d'une figure. Le dispositif technique occupe une place centrale dans l'espace et le jeu des interprètes relève en grande part du geste technique : leur geste d'animation consiste justement à faire apparaître des images.

Rêves et motifs s'est construit à partir du recueil de textes inédits du mathématicien Alexandre Grothendieck (1928-2014), Récoltes et semailles ${ }^{19}$. La multiplication des dispositifs de projection mis en place par les Rémouleurs en divers endroits du plateau et de la salle (camera lucida, rétroprojection, jeux d'ombres produits par les mouvements de flammes de bougies, ...) reflète l'hétérogénéité des textes rassemblés dans cet ouvrage (textes théoriques sur les mathématiques, souvenirs d'enfance, considérations politiques...). La dispersion des sources lumineuses, combinée à la diversité des surfaces de projection, créent l'effet d'une omniprésence et d'une démultiplication de la figure. Celle-ci se manifeste dans le visible sous des formes changeantes et toujours évanescentes mais qui envahissent progressivement l'ensemble de la salle. La spatialisation de la figure tient ainsi à la mise en constellation de ses manifestations visibles. Dans le cas de Rêves et motifs, elle évite le dessin figé d'un personnage pour mieux traduire une pensée en mouvement et une présence ancrée dans l'actuel, quoique convoquée depuis un proche passé.

Par ailleurs, le miroir liquide au centre du plateau donne à voir des irisations changeantes à la surface d'un film de savon. Cette surface est extrêmement transparente et pourtant capable de réflexion. Elle agit donc à la fois comme miroir et comme filtre déformant pour les spectateur.trice's située's de part et d'autre de la structure. Le miroir peut être traversé par une main sans se briser, faisant apparaître ainsi des images de corps siamois et fragmentaires. L'extrême fragilité de cet écran confère un caractère volatile aux images quasi-abstraites qui s'y forment.

Un tel travail sur la matérialité des écrans se rencontre dans toutes les créations de la compagnie Les Rémouleurs. Nous le trouvons dans L'Oiseau (2016) avec ce gigantesque oiseau en vol sur lequel sont projetées des images [Figure 9] ou dans Freaks (2013), qui se joue dans les cafés et les bars et où le jeu des projections transforme des espaces quotidiens en redessinant leurs volumes grâce à des projections d'images empruntées à l'imaginaire forain. Dans la pratique des Rémouleurs, l'image est par excellence le lieu de la recherche plastique et de l'animation. Son caractère impondérable place au cœur de la dramaturgie la question de l'apparaître et de la persistance de la présence. Le devenir-image ${ }^{20}$ de la figure marionnettique lui confère un caractère d'omniprésence conjugué paradoxalement à une grande fragilité. Les expériences des Rémouleurs reposent ainsi essentiellement sur la subtilité matérielle des figures qu'ilelle's font 
apparaître, en équilibre vacillant entre matérialité et immatérialité. Une telle démarche ouvre sur des présences marionnettiques qui déborde le champ du visible et de la matière pour acquérir un caractère ondulatoire, parent du flux lumineux.

Figure 9

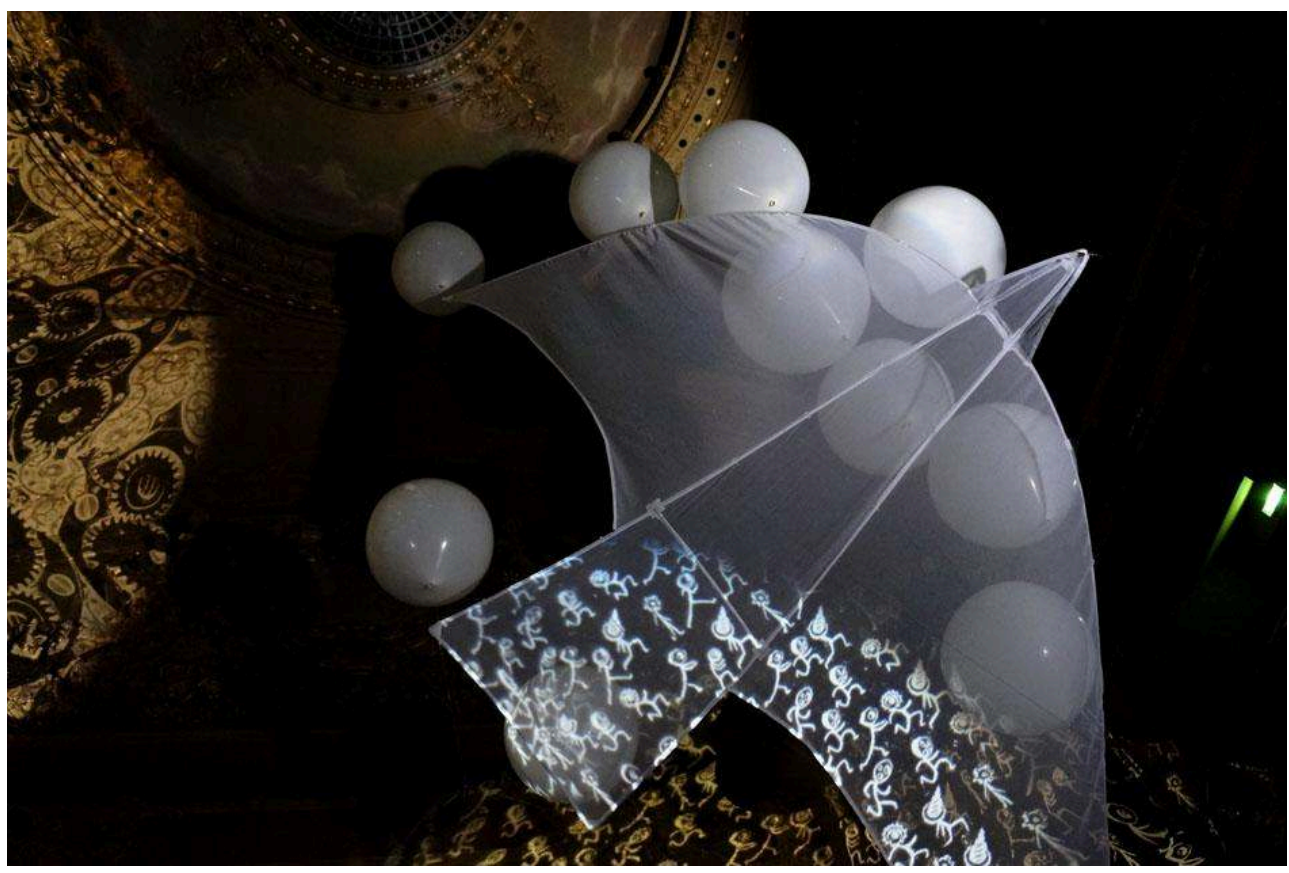

L'Oiseau, Les Rémouleurs, 2016 (Photo : Gallia Vallet)

\section{De lumières et de sons : vers la marionnette ondulatoire}

La manipulation de la lumière permet d'animer l'immatériel et l'informe, de sculpter l'espace autour des corps, de stratifier l'espace avant l'écran. Chez Gisèle Vienne et Benjamin Verdonck, la défection de l'objet-marionnette passe également par une approche plastique de la lumière, qui à la fois défait les limites matérielles des objets visibles et dynamise l'espace entre eux. Ainsi, la lumière, comme médium ondulatoire, participe pleinement de l'élaboration des présences marionnettiques - au même titre que les corps et les objets visibles-bien qu'elle produise des figures davantage impondérables.

Gisèle Vienne s'attache dès les premières résidences d'un projet à la création de la lumière. Elle considère « le texte, la lumière, la musique, comme d'autres interprètes, tout simplement ${ }^{21}$ ». Le principe de l'animation repose selon elle sur le fait de «"laisser la parole à", laisser la parole aux autres choses, qui ne sont pas humaines ${ }^{22} »$ :

Ça peut concerner un objet qui rappellerait la marionnette ou tout autre objet. Pour moi, c'est tout aussi important à l'égard de la lumière ou de la musique. À certains moments, il s'agit de laisser la parole de manière très explicite à la lumière ou au son. ${ }^{23}$

Une telle approche de la figure, notamment construite par la lumière, nourrit notre conception d'une marionnette immatérielle.

Cette approche hérite des fantasmagories du XIX ${ }^{\mathrm{e}}$ siècle autant que des expériences scéniques symbolistes. Cristina Grazioli, chercheuse italienne en esthétique et histoire 
du théâtre, s'intéresse notamment à la lumière dans la perspective de son lien avec les pratiques marionnettiques. Elle parle ainsi d'« acteurs de lumière " pour désigner «cette grande famille de figures qui [...] prennent forme grâce à la lumière ou à l'ombre $^{24}$ ». Elle trace leur filiation des «premières images de lanterne magique ${ }^{25}$ » au théâtre d'ombre contemporain, en passant par l'ombromanie, les "présences fantomatiques des "genres optiques" 26 " et "l'univers infini de toutes les traditions d'ombre, plus ou moins immatérialisé, plus ou moins "ombreux" 27 ». Cristina Grazioli considère le poète et dramaturge Rainer Maria Rilke comme un penseur majeur de ces présences lumineuses. Elle évoque, à propos des personnages de ses pièces, des figures évanescentes " "trempées" dans la lumière, une lumière éblouissant notre regard ${ }^{28}$ ". Nous retenons de ce terme "éblouissant » la double désignation, presque paradoxale, d'une très grande clarté et d'une saturation sensorielle, confinant à l'aveuglement. Les figures dont la forme n'apparaît que par la sculpture de la lumière instillent en fait le doute sur leur existence réelle. Le symboliste Maurice Maeterlinck avait lui-même pressenti la possible substitution d'" une ombre, un reflet, une projection de formes symboliques $^{29}$ » au corps de l'interprète humain.

Figure 10

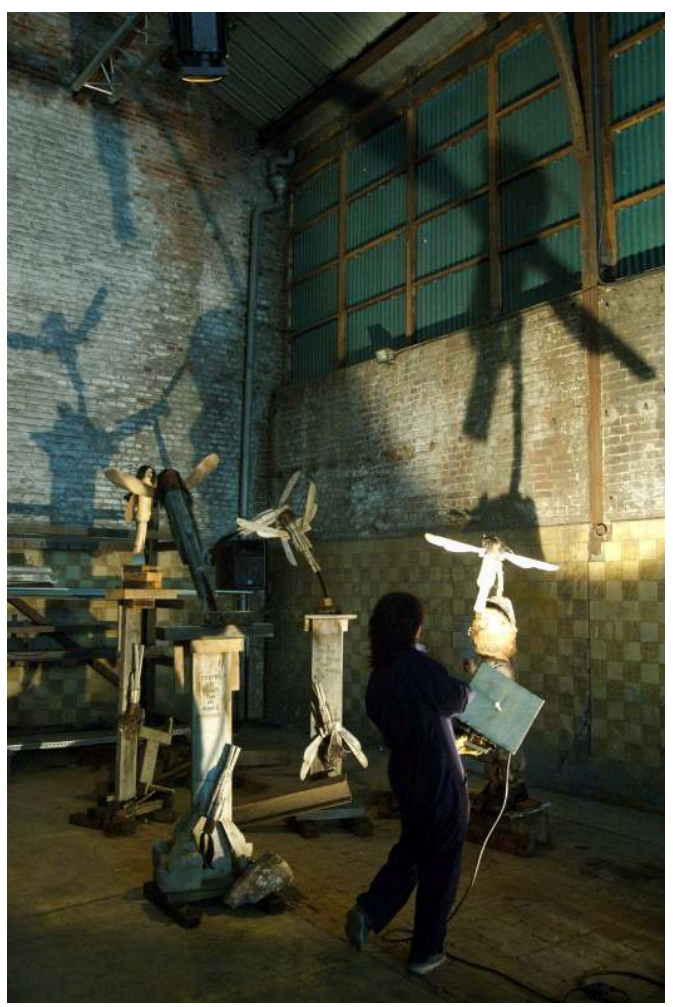

Des hurlements montaient le long des saules pleureurs, Clastic Théâtre, 2013 (Photo : Clastic Théâtre Sculptures : Anges, Francis Marshall)

Aussi le travail de la lumière est fondateur des expériences esthétiques contemporaines sur les présences infigurées. La filiation historique nous rappelle que le théâtre est depuis longtemps un lieu de prédilection pour l'édification de présences marginales, non exactement humaines, à la densité fantomatique. En effet, malgré la grande diversité esthétique des créations contemporaines qui retiennent notre attention, nous y repérons un certain nombre de techniques transversales à ces écritures de l'infiguré. 
Le jeu d'ombres [Figure 10], la vaporisation de brouillards, les effets de réflexion lumineuse, l'utilisation de tulle [Figure 11] et l'utilisation de flash lumineux très intenses permettent, par action sur l'intensité et la direction de la lumière, la multiplication, l'effacement ou la déformation des contours des corps.

Figure 11

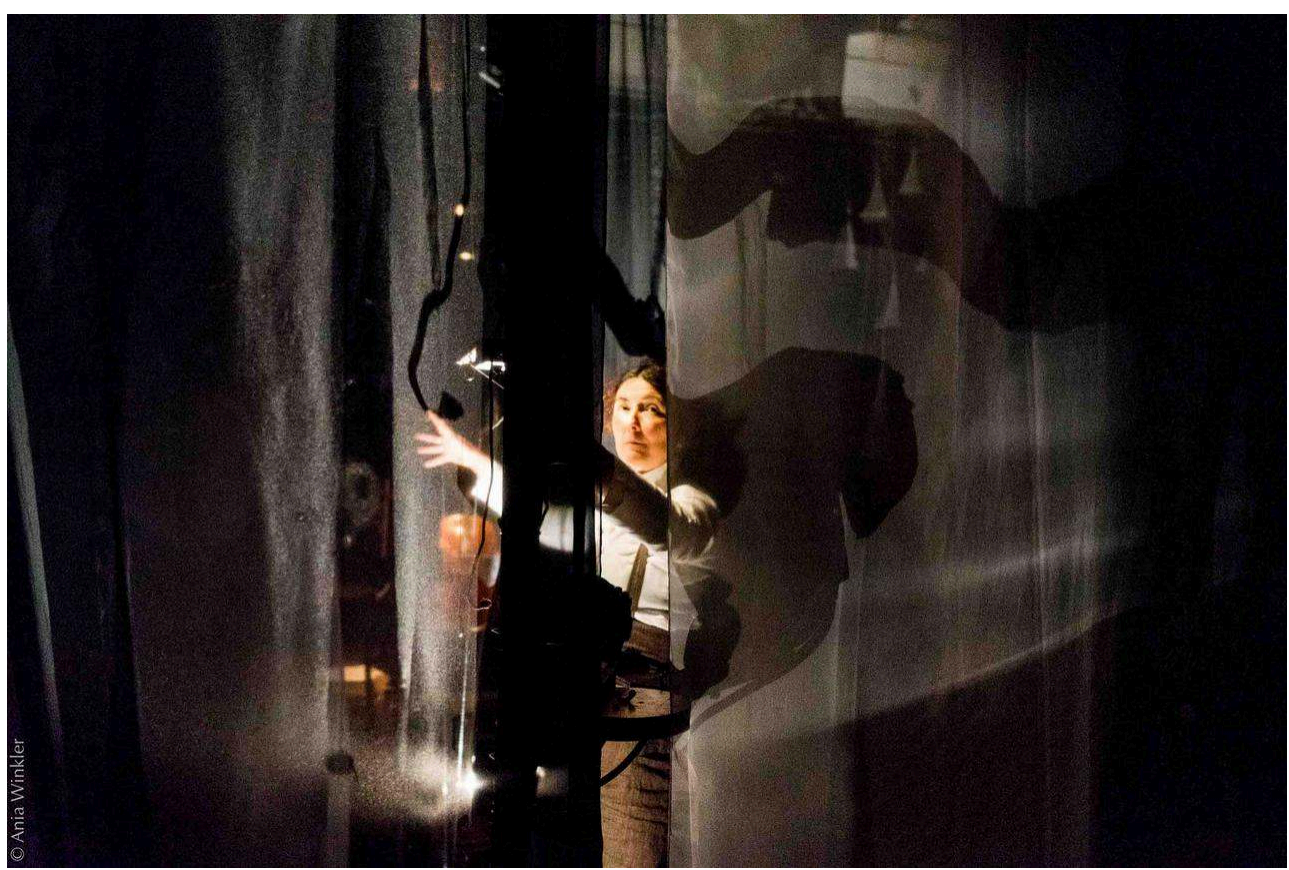

Rêves et motifs, Les Rémouleurs, 2017 (Photo : Ania Winkler)

Pourtant, la particularité des créations contemporaines qui retiennent notre attention est de mettre en scène ces processus comme en cours. Autrement dit, alors que les fantasmagories romantiques ou les expériences symbolistes cherchaient à manifester des présences appartenant à un autre degré de réalité, les figures marionnettiques contemporaines ne s'ancrent pas clairement dans un ailleurs: elles creusent à vue l'écart entre la forme visible et la présence tangible. Ces écritures mettent en scène une dégradation de la qualité incarnée des figures. La défiguration en procès devient ellemême l'objet du drame.

Pour sa création The Pyre en 2013, la metteuse en scène Gisèle Vienne choisit de ne plus travailler qu'avec des danseur'se's et de se passer des mannequins réalistes qui marquaient l'esthétique de ses précédentes créations. Pourtant le dispositif lumineux et sonore dans lequel sont placée's les interprètes éveille ponctuellement le doute sur leur caractère humain. Dans la scène inaugurale de cette création, la danseuse Anja Röttgerkamp apparaît quasi-immobile, dans une pose désarticulée, au centre d'un dispositif lumineux et sonore qui semble se déployer et se mouvoir indépendamment, et autour d'elle. Grâce à une technologie de spatialisation du son (Spat) et à l'utilisation de deux murs de LEDs, l'espace scénique apparaît tantôt très resserré, tantôt très ouvert, selon que seul le bas de la scène est éclairé ou que quelques LEDs éparses dessinent des étoiles sur un fond noir. Cet effet de variation du volume de l'espace donne à voir un corps humain qui ne maîtrise pas mais subit ses déplacements et immobilisations. Gisèle Vienne a également choisi un sol en tapis de danse hyperréfléchissant qui défait le contour net de la danseuse. Au cours de la représentation, le 
corps d'Anja Röttgerkamp apparaît donc tour à tour comme l'image incertaine et évanescente d'un corps féminin, ou comme un corps habité d'une présence humaine plus réaliste. La singularité dramatique de ce corps tient à son oscillation permanente entre une densité incarnée et celle, inquiétante, d'un corps spectral. Une telle oscillation raconte dans The Pyre à la fois la violence subie par une femme et le travail mémoriel d'un enfant cherchant à convoquer le spectre de sa mère décédée.

\section{Animation de l'espace « entre » les corps}

Si les enjeux dramaturgiques diffèrent d'une création à l'autre, le travail mené par Gisèle Vienne rencontre celui de Benjamin Verdonck ou encore celui des Rémouleurs à l'endroit de ce travail plastique de la lumière qui confine à l'illusion d'optique. Il tend en effet à l'animation illusoire des espaces vides autour des formes, des espaces relationnels entre les corps et les objets.

Benjamin Verdonck a travaillé dès l'origine du projet Chansonnette pour Gigi avec le créateur lumière Lucas Van Haesbroeck, spécialiste des illusions d'optique. L'enjeu de leur collaboration est de manipuler les automatismes optiques des spectateur.trice-s mécanismes de reconnaissance des mouvements dans l'espace, de lecture des distances - pour leur faire apparaître des formes qui n'ont pas de matérialité propre et pour produire des mouvements illusoires. L'impression de voir un rectangle sombre en lévitation dans un cadre vide, la sensation d'un espace qui se resserre ou de plans qui avancent et reculent peuvent naître de la simple variation de l'intensité lumineuse et de la répartition des ombres sur les plans verticaux du castelet animé [Figure 12]. Les spectateur.trice's sont ainsi placé'e's en position de voir ce qui n'est pas et, le sachant ${ }^{30}$, ils acceptent l'incertitude permanente sur la réalité des formes visibles comme une invitation à la rêverie.

Figure 12

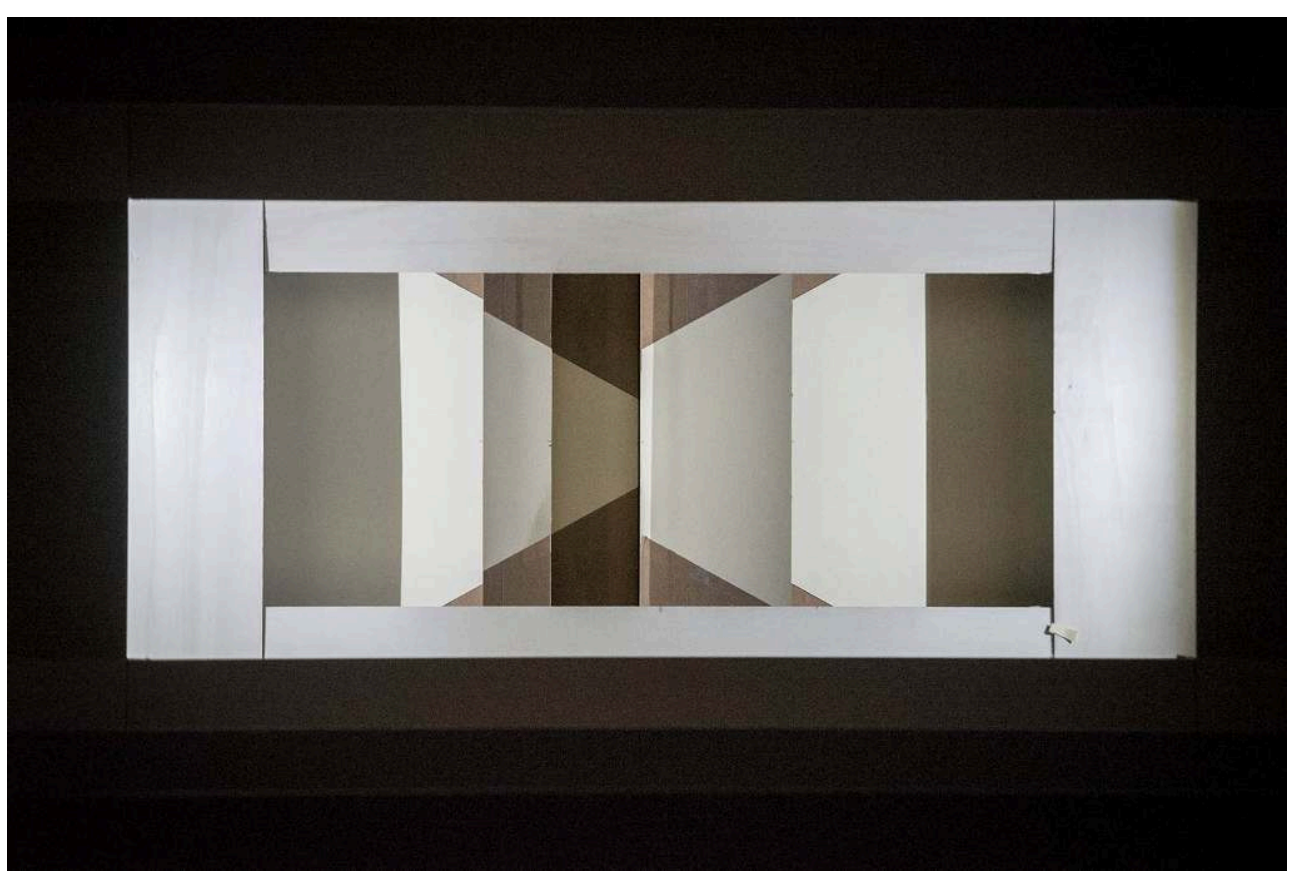

Chansonnette pour Gigi, Benjamin Verdonck, 2018 (Photo : Kurt van der Elst) 
Benjamin Verdonck réalise très concrètement l'animation de l'espace "entre " les corps lorsqu'il n'offre plus aux regards que des cadres ouvrant sur des espaces vides. Seule l'ouverture des cadres en leur centre et les variations lumineuses créent l'effet d'un espace travaillé par des présences invisibles. Le texte dit et la musique participent également à la projection très libre de figures dans cet espace qui évoque l'espace intime de la maison. La façon dont les lumières rencontrent les planches verticales qui découpent en strates l'espace du grand castelet de bois évoque d'ailleurs les intérieurs peints par Vihelm Hammershøi (1864-1916). Or, Cristina Grazioli nous rappelle que Rainer Maria Rilke, dont nous évoquions précédemment les figures dramatiques évanescentes, était lui-même admiratif des œuvres du peintre danois, peintre des "figures imprégnées de lumière, des intérieurs marqués par des seuils et par des relations entre les présences et l'espace ${ }^{31}$ " [Figure 13]. Ainsi nous reconnaissons dans les écritures de la marionnette infigurée un lien entre le travail de la lumière, la spectralisation des corps et l'élaboration d'un espace habité, que nous désignons aussi comme effet de spatialisation de la marionnette infigurée.

Figure 13

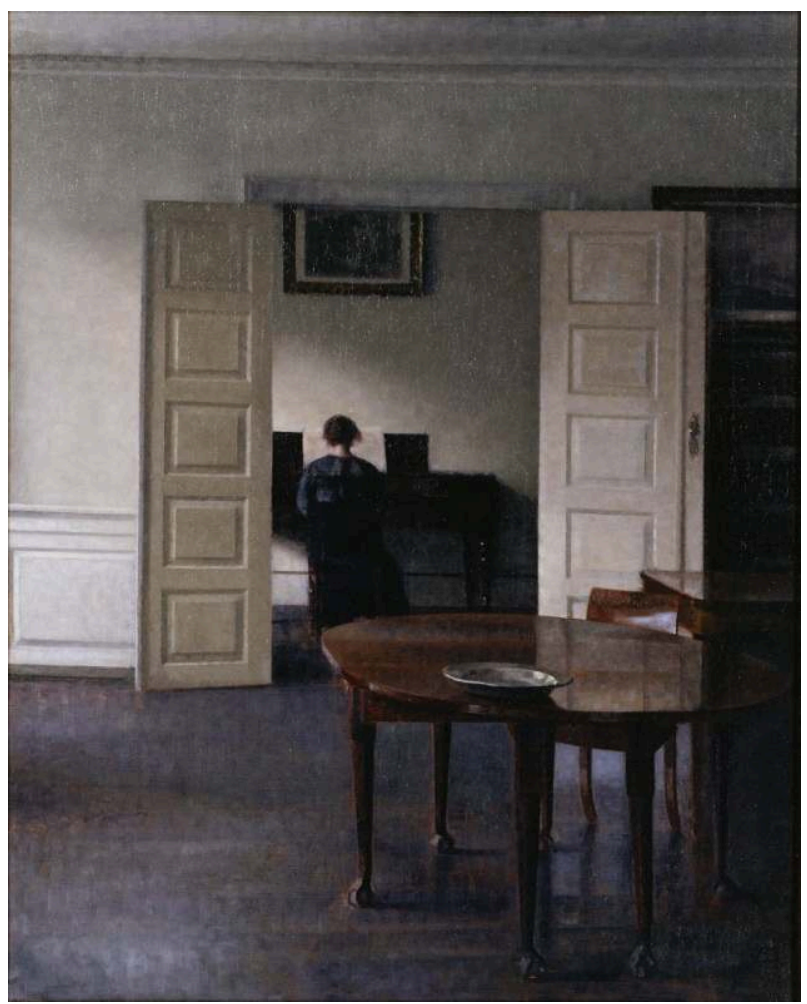

Intérieur avec Ida jouant du piano, Vilhelm Hammershøi, 1910

Ouvrant sur un horizon vide, au " creu[x] de la matière ${ }^{32}$ ", l'espace marionnettique est stratifié et rendu sensiblement dynamique par les médiums ondulatoires qui le traversent. La sculpture de l'espace par ces flux immatériels concourt à l'obsolescence de l'objet-marionnette. L'effet d'une présence circulant à travers l'ensemble du dispositif ouvre en effet sur une conception cinétique, illusoire et non matérielle de la marionnette. La "scène ondulatoire ${ }^{33}$ " qu'Antonin Artaud rencontre dans le théâtre balinais n'est pas si lointaine d'une telle conception de l'écriture marionnettique ${ }^{34}$. 
Dans la lecture qu'en fait Artaud, la danse, la spatialisation du son et le mouvement des lumières confèrent à l'espace une physicalité paradoxale :

Les guerriers entrent dans la forêt mentale avec des roulements de peur, un immense tressaillement, une volumineuse rotation comme magnétique s'empare d'eux, où l'on sent que se précipitent des météores animaux et minéraux.

C'est plus qu'une tempête physique, c'est un concassement d'esprit que le tremblement épars de leurs membres et de leurs yeux roulants signifie. La fréquence sonore de leur tête hérissée est par moments atroce, - et cette musique derrière eux qui se balance en même temps alimente on ne sait trop quel espace où des cailloux physiques finissent de rouler. ${ }^{35}$

Le témoignage d'Artaud éclaire la façon dont le geste - souvent dansé - et la musique persistent, dans les créations contemporaines, à faire signe vers des présences alentour quoique celles-ci ne prennent pas forme. Une forme de vibration générale, qualifiée de "tressaillement» puis de «tremblement», traverse l'espace scénique. Comme on le reconnaît dans Chansonnette pour Gigi ou encore dans les Hurlements du Clastic Théâtre, l'espace en vibration acquiert une organicité et un dynamisme, grâce auxquels des figures peuvent fugacement advenir, devenir sensibles aux spectateur.trice's, quoique ne possédant pas de corps.

De la dégradation de l'objet à la mise en tension de l'espace "entre ", en passant par l'évidement des corps et la mise en scène de l'informe, les processus sont donc multiples, qui mettent en échec le principe d'objet-marionnette, entendu comme l'association d'un corps matériel identifiable à la manifestation tangible d'un sujet dramatique. Dans les créations contemporaines, la matière ne joue plus sur le mode métaphorique. Elle n'a pas pour fonction de donner figure. Elle est davantage le témoin d'un mouvement. Permettant l'apparition fugace d'une forme, elle devient le symptôme d'une présence marionnettique (c'est-à-dire non-incarnée). La figure circule ainsi non plus seulement en-dehors du corps humain mais plus globalement en-dehors des formes visibles et des limites de la matière. En ce sens, les approches dramatiques de François Lazaro, de Benjamin Verdonck, des Rémouleurs et de Gisèle Vienne fondent des drames de l'infiguration. Ces artistes nourrissent de ces figures périssables et incertaines des dramaturgies du rêve, de la folie ou de la mémoire, qui placent le doute et l'incertitude au cœur des processus de réception. Ces figures parviennent à rendre sensibles les invisibles qui nous hantent.

Une fois affirmée cette tension vers l'infiguré, il importe pourtant de revenir sur la place prépondérante qu'occupent les objets dans les créations en question. L'identification d'une figure marionnettique ondulatoire n'est pas synonyme de dématérialisation générale des plateaux. Il nous faut prêter attention à la fonction spécifique de la matière, là où elle persiste dans ces créations qui renouvellent le drame marionnettique.

\section{Persistance de la matière}

Si elles mettent en jeu des marionnettes infigurées, les scènes des Rémouleurs, de Gisèle Vienne, de Benjamin Verdonck et du Clastic Théâtre, sont loin d'être tout à fait vides. Elles accordent une place fondamentale dans l'économie des présences à des objets, des outils, des machines, des matières, tout autant que le travail à l'atelier occupe souvent une place centrale dans les processus de création. Il apparait donc que si la figure marionnettique se singularise par sa présence possiblement défigurée, la 
spécificité des écritures marionnettiques continue d'être du côté d'une mise en jeu singulière des matières et du geste plastique. Déviant de la fonction métaphorique de l'objet-marionnette, la matière agit dans les créations qui nous intéressent dans un sens davantage phénoménologique : c'est sur son «être-là », ses apparitions, expansions, disparitions, ou son irréductible existence physique que se fonde son potentiel dramatique.

\section{Oscillations de la figure virtuelle, ambivalences du numérique}

La dématérialisation de la figure marionnettique pourrait être mise en regard du déploiement actuel des technologies numériques dans les arts dramatiques. Nous pourrions faire l'hypothèse d'une influence des nouveaux médiums numériques sur la qualité impondérable de la figure marionnettique. L'utilisation de logiciels de captations du mouvement, de mapping, de projections tridimensionnelles, de spatialisation du son, a en effet entrainé "les artistes, comme le note Florence de Mèredieu dans son ouvrage Arts et nouvelles technologies, dans la voie d'un immatérialisme de plus en plus radical ${ }^{36}$ ». L'universitaire observe, en des termes qui font particulièrement écho à l'histoire de l'objet-marionnette, que

l'œuvre s'allège de ces supports « lourds » que sont le bois, le marbre, le châssis et le cadre du tableau, etc. Ce qui constitue le support de l'œuvre, c'est, de plus en plus, l'« image »- fluidique, immatérielle, en apesanteur. L'image désincarnée des nouveaux médias $[\text { sic }]^{37}$

Nous observons à notre tour que les créations qui font l'objet de la présente étude recourent à des logiciels de montage et de spatialisation du son, tels Ableton Live ou la technologie Spat ${ }^{38}$. D'autres outils numériques permettent l'écriture des lumières et la création de partitions lumineuses particulièrement subtiles et précises.

Pour autant, nous remarquons aussi dans ces créations l'absence d'images numériques en tant que telles. Soit les figures virtuelles sont créées sans le recours numérique, et marquées du sceau de la main humaine et artisane, soit on observe une forme de résistance à l'élaboration de figures simplement virtuelles: le numérique, lorsqu'il participe à l'élaboration des présences, est mis en jeu de façon à problématiser en fait le caractère virtuel ou l'absence des figures.

Les Hurlements du Clastic Théâtre illustre le premier cas: les projecteurs qui créent l'effet d'un mouvement général de l'espace sont manipulés en prise directe par les interprètes et l'ensemble de la musique est acoustique, réalisée par deux interprètes qui utilisent entre autres les objets directement trouvés dans la forge pour créer un univers sonore propre au lieu. Dans Chansonnette pour Gigi, la musique est également interprétée en direct par deux musiciens visibles à l'avant-scène, et Benjamin Verdonck occupe une position en retrait sur le côté du castelet, mais reste visible dans son rôle de manipulateur et artisan des illusions auxquelles assiste le public. Olivier Vallet des Rémouleurs n'utilise lui non plus aucune technologie numérique de production d'images : il considère comme «froides » les images numériques et apprécie les images non-pixellisées et sans cadre produites par le seul jeu des lentilles dans les camera lucida et les rétroprojecteurs ${ }^{39}$.

Dans The Pyre de Gisèle Vienne, le numérique est clairement thématisé dans le texte de Dennis Cooper (qui évoque l'isolement d'un adolescent errant sur des forums de chat), quand la scénographie et la qualité des corps en scène rappellent les esthétiques numériques, très lisses, lumineuses, aériennes. Pourtant, le traitement des corps des 
danseur'se's, leur éclairage et leurs gestuelles les rappellent aussi ponctuellement à leur dimension physique et incarnée.

Ainsi l'ancrage dans la matière et le corps importent dans ces créations en tant que contrepoint à l'élaboration de présences infigurées. Les figures ondulatoires que nous rencontrons sur les plateaux de ces artistes marionnettistes ne sont donc pas assimilables à des figures numériques. Quelque chose d'une persistance du corps et de la matière passe dans la création artisanale, en direct, de figures évanescentes. Nos regards, aujourd'hui rompus aux images numériques, continuent d'être sensibles à la trace laissée par un corps véritable sur des simulacres. La main humaine, la dimension charnelle d'un corps, restent apparents dans ces créations, même sous la seule forme d'un geste passé. Francis Marshall laisse apparent les traces de son bricolage. Gisèle Vienne, inspirée des portraits de Fernand Khnopff, laisse apparentes les traces du pinceau sur les visages des mannequins d'I apologize.

Ainsi, toutes ces créations, en accordant une place paradoxale au corps absent problématisent la qualité des figures et le lien entre présence, corps et matière. La question matérielle est en fait au cœur de ces dramaturgies et la visibilité du geste plastique ouvre une réflexion sur les supports de l'image. Olivier Vallet, de la compagnie Les Rémouleurs, explique par exemple que dans leur précédent spectacle, Boucle d'or (2010), le film du miroir liquide était assez résistant pour ne pas se briser. Les artistes avaient alors choisi de le briser volontairement afin de rendre perceptible la matérialité singulière de cet écran. L'enjeu était d'éviter qu'il ne fonctionne comme simple médium transparent des images qui s'y formaient. Pour les Rémouleurs, ce type d'images animées constitue un contrepoint poétique aux images numériques auxquelles nos regards sont perpétuellement confrontés. L'inconstance de leur épaisseur matérielle permet un doute critique sur la réalité des formes. Les caractéristiques physiques des médiums choisis permettent d'interroger notre rapport au visible. Faute de donner forme et corps à une figure stable, les matières résistent en cela à la simple métaphorisation. En termes médiologiques, les matières discontinûment animées fonctionnent comme médiums alternativement transparents et opaques.

\section{Opacité, résistance, et persistance de la matière}

L'approche poétique de l'opacité de la matière consiste à envisager davantage son êtrelà phénoménologique que son pouvoir de figuration. Elle permet ainsi la mise en jeu d'une potentielle résistance à la manipulation ${ }^{40}$. La résistance de la matière est un tenant du drame d'I apologize de Gisèle Vienne, qui emploie elle-même l'expression de "résistance passive ${ }^{41}$ » des objets. Lorsqu'ils sont discontinûment manipulés, ses mannequins produisent l'effet d'une désanimation. Ils donnent ainsi ponctuellement l'illusion de résister à leur manipulation, opposant à l'interprète-narrateur (incarné par Jonathan Capdevielle) leur poids et leur inertie propre. Dans cette création, le personnage principal a un rôle de narrateur ou metteur en scène, cherchant à figurer ses fantasmes érotiques et meurtriers. Or, la volonté de ce personnage est aux prises avec des corps et des objets qui oscillent entre malléabilité et résistance, soumission et rébellion. En effet, si différents éléments esthétiques signifient la soumission des corps à la volonté fantasmatique - visages et paupières baissés, station irréductiblement assise ou allongée, manipulation en prise directe, immobilité dont sourd une forme d'attente et d'obéissance - ces objets font aussi voir ponctuellement une opposition aux 
désirs de figuration du manipulateur. Par leur inertie et leur rigidité non-organique, les mannequins paraissent parfois s'opposer à la réalisation de l'image et aux mouvements que veut leur imprimer le manipulateur. La manipulation très précise de Jonathan Capdevielle, marionnettiste formé à l'ESNAM, joue sur deux plans : un premier, visible, qui fait voir l'effort de son personnage pour imprimer des postures torturées aux poupées ; un second, plus dissimulé, qui imprime aux objets des mouvements fugaces et minuscules d'opposition à ses poussées. Ce jeu crée l'impression que les poupées cherchent à échapper à sa volonté dominatrice, en chutant ou en se raidissant. L'effet de résistance passive tient donc à un geste manipulatoire situé en-deçà de l'animation et de l'imitation du mouvement vivant. Par ailleurs, la construction de ces objets leur permet aussi cette forme de résistance. Gisèle Vienne opte pour des matériaux offrant un équilibre entre rigidité et souplesse, nécessaire à ces effets. De même la construction de leurs articulations (épaules, coudes, hanches, cou) est orientée par cette recherche de très subtile résistance. Le travail manipulatoire et plastique vise donc le surlignement ponctuel de la nature d'objets des mannequins mis en scène. C'est ce que nous nommons l'opacification des objets scéniques. Enfin, la résistance passive des mannequins passe par leur nombre. Lorsqu'ils jonchent le sol [Figure 14], les objets occupent physiquement l'espace, ils s'y ancrent. Ces corps résistent en ce qu'ils se constituent en traces ineffaçables du crime (commis ou rêvé), en corps irrémédiablement présents. Ces mannequins-qu'il serait imprécis de qualifier d'objets-marionnettes, parce qu'ils ne se constituent jamais sûrement en corps de sujets dramatiques, et parce que leur animation est toute relative - acquièrent grâce à leur nombre, leur construction et leur manipulation spécifique, le pouvoir de signifier en tant qu'objets, par leur inertie, leur poids, leur rigidité non-organique. Leur condition dramatique est précisément de ne pouvoir disparaitre ou s'effacer. Une telle écriture, avec la matière, d'un conflit entre l'humain et les objets qui l'entourent, dramatise les rapports à l'autre déshumanisant, les désirs de maîtrise et la résistance du réel au fantasmatique. 
Figure 14

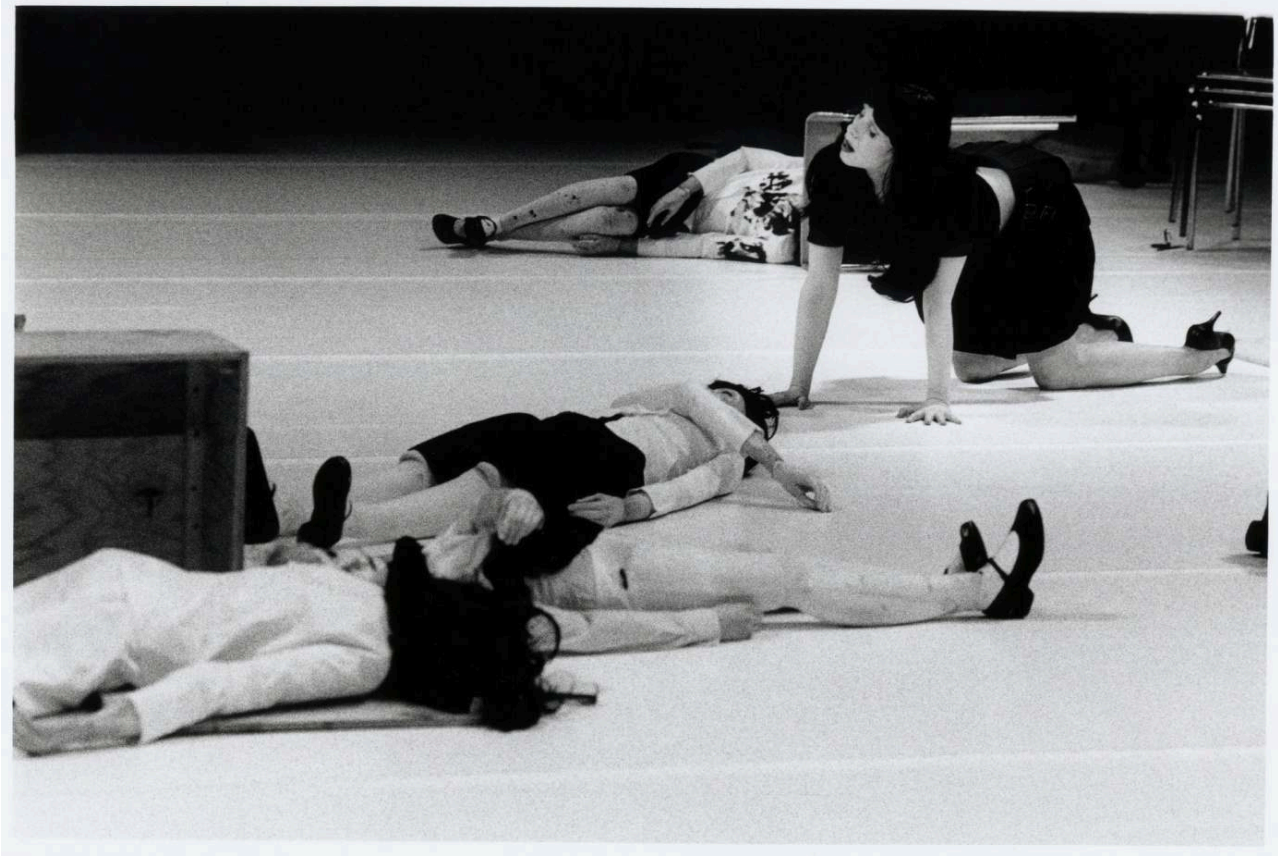

I apologize, Gisèle Vienne, 2004 (Photo : Mathilde Darel. Interprète sur la photo : Anja Röttgerkamp)

La résistance passive de la matière, qui tient à son seul être-là dramatisé, acquiert le sens poétique d'une "persistance ", dans la création des Hurlements du Clastic Théâtre. L'écriture de François Lazaro confère aux machines de la forge ardennaise un statut de matière "qui reste " malgré l'abandon de la main humaine. Faute de fonctionner en objets-marionnettes, ces objets sont érigés, par leur inertie même, en témoins d'une disparition en cours. Les représentations des Hurlements ont lieu dans l'usine ardennaise à la tombée de la nuit, c'est-à-dire lorsque les travailleur-se's en sont absente's et que les machines sont à l'arrêt. Or, cette absence de mouvement réel des instruments de travail est éloquente. Alors que la vue des machines renvoie à un savoir-faire technique et au geste ouvrier, la main humaine n'intervient pas dans le spectacle pour manipuler ces outils temporairement abandonnés. Alors qu'en 2013, lors des représentations, la forge de Nouzonville est la dernière forge encore en activité dans les Ardennes, les objets inanimés y sont gigantesques et présents en nombre. Ils font preuve ainsi d'une persistance là où la main humaine a déjà en partie disparu. Ces objets immobiles concourent ainsi à l'édification de la forge en figure de survivante. Cette exhibition de la machine qui ne fonctionne pas faute d'humains rappelle la démarche de Nam June Paik, artiste du mouvement Fluxus, qui créa en 1965 le premier androïde : «en réaction aux scénarios prédisant que les machines supprimeraient les emplois des hommes ", l'androïde K456 qu'il construisit, " nécessitait l'intervention concomitante de cinq opérateurs humains pour pouvoir fonctionner ${ }^{42} »$. Les machines de la forge, à la façon de cet androïde, formulent en creux l'absence de la main humaine, qui a disparu des nombreuses autres usines de la région. La forge apparaît ainsi comme une trace matérielle en suspens, subsistant, en suspens après la disparition.

Entre ces machines immobiles et les espaces de stockage et de rangement qui s'élèvent jusqu'aux plafonds de l'usine, François Lazaro installe les pantins de Francis Marshall, faits de matériaux usés et mesurant environ un mètre de haut. Sans que ni les engins 
métalliques, ni les objets de tissus ne fassent l'objet d'une manipulation concrète, le très fort contraste entre eux crée un dialogue spectaculaire des matières. D'une part, les écarts de tailles rendent criant le caractère fragile et l'impuissance des pantins anthropomorphes. D'autre part, leurs corps en voie de délitement, face aux lourdes machines de fer, font apparaitre une très grande précarité. Leurs postures avachies contrastent avec la hauteur des espaces de l'usine. Ce jeu de confrontation des extrêmes érige dramatiquement le dur contre le mou, l'éphémère contre le durable, le minuscule contre le gigantesque. Le contraste plastique fait écho à un conflit d'actualité : l'état de délabrement matériel des pantins anthropomorphes soutient un dialogue virtuel avec la forge. À travers lui, se structure un drame de l'humain aux prises avec l'usine et, plus largement, avec les dommages du capitalisme. Les matières sculptées par Francis Marshall restent malgré tout et témoignent d'une fin imminente. Ces objets pauvres évoluent, comme l'écrit Jean-Luc Mattéoli, « dans cette couche de temps fantomale de l'à-peine-passé qui est celle privilégiée par la mémoire ${ }^{43}$ ». Ils sont "dans une époque monstrueuse, un témoin de la disparition des êtres et des choses, vécue sur le mode de la perte ${ }^{44} »$. Le motif de l'inquiétude tient en effet à une part d'absence, que le dialogue des matières - en voie de délitement, d'abandon, et qui pourtant persistent - manifestent déjà en puissance.

Les caractéristiques matérielles des objets des Hurlements valent dramaturgiquement autant que les formes qu'ils donnent à voir. Dans ce trouble du fonctionnement iconologique des objets, la matière persistante acquiert un nouveau statut dramatique : elle « ne montre rien, mais donne à saisir le vestige homonyme d'une absence ${ }^{45} »$. Cette faculté de manifestation d'une absence relève d'un fonctionnement dramaturgique en négatif par rapport à celui d'un objet-marionnette, qui actualiserait la présence d'un sujet fictif.

Montrer des objets immobiles, résistants au mouvement, a donc pour effet de déplacer la fonction dramaturgique vers les marges de la manipulation marionnettique. La discontinuité des procédés d'animation fait voir alternativement la matière pour ce qu'elle est. La matière se met à signifier par son inertie et sa composition plastique plus que par son mouvement et sa forme. Cette monstration de la matière ouvre sur une interprétation critique et potentiellement politique de la persistance des objets : dans les Hurlements, l'objet est ce qui ne s'efface pas quand tout a disparu. Dans l'ombre, mais gigantesques, les outils rangés et les machines à l'arrêt de la forge de Nouzonville «SONT »- pour reprendre la formule de Tadeusz Kantor, qui affirmait que «l'objet EST tout simplement ${ }^{46} »$. La graphie en majuscules traduit la matérialité incompressible de l'objet, qui agit comme obstacle à la disparition et l'oubli.

\section{Vers une visibilité minimale : des balises pour le regard}

Dans le procès de dématérialisation de la figure, écrire à partir de la matière l'élaboration de figures impondérables constitue la spécificité persistante de la création marionnettique. La cartographie du visible et de l'invisible repose sur une approche des médiums-matériaux comme balises pour le regard. Ils cadrent le cheminement des spectateur-trice's vers l'infiguré. Benjamin Verdonck conçoit ainsi le dispositif de Chansonnette pour Gigi comme un outil de décrochage par rapport au réel. La berceuse, évoquée dès le titre et dont la teinte se retrouve dans la musique comme dans la diction du texte, occupe précisément cette fonction d'invitation à la méditation et au rêve. Avec Chansonnette pour Gigi, Benjamin Verdonck renonce aux triangles qu'il manipulait 
dans Notallwhowanderarelost. Seul le mouvement de rectangles de bois les constitue en scénographie miniature et animée, indiquant au regard non pas de les regarder mais où regarder. Ces formes géométriques aux mouvements minimaux nous orientent vers le creux profond du castelet animé, nous indiquant un cheminement vers des présences infigurées.

Sur le plan esthétique, de tels mécanismes d'infiguration proposent un décalage de nos habitudes de regard. Ils agissent à contre-courant de ce que Marie-José Mondzain, philosophe de l'image, nomme « l'empire des visibilités ${ }^{47}$ ». Opposant conceptuellement le visible et l'image, elle affirme qu' « il y a de moins en moins d'images. Les formes les plus récentes des visibilités donnent l'impression d'une inflation sans précédent du flux iconique [...] Le débordement des visibilités fait peser sur le destin de l'image et, par voie de conséquence, sur la responsabilité du regard une vraie menace ${ }^{48}$ ». À la lumière de ces propos, nous reconnaissons que l'évolution du statut dramaturgique de la matière dans les écritures marionnettiques pourrait fonder une attitude spectatorielle davantage critique et émancipée : l'image se dérobe au visible mais la matière agissant sur le mode non-métaphorique, non-iconique, propose aux spectateur-trice's un cheminement sensible. Il invite à un travail imaginaire, sans lequel ne se joue pas de drame infiguré. En d'autres termes, la puissance dramatique de ces dispositifs, qui creusent le visible, tient à une organisation du sensible dans laquelle la matière et son mouvement occupent, même en négatif, une fonction primordiale, celle de pointer vers des présences, de les indiquer, plus que de les incorporer.

\section{Conditions matérielles de production de figures impondérables}

Finalement, c'est dans une perspective pratique et politique qu'il importe de souligner la persistance de la question matérielle dans les créations marionnettiques. Malgré la dématérialisation de la figure, la matière endosse dans ces écritures un rôle singulier qui confronte les artistes à des problématiques propres aux champs des arts de la marionnette. Notre insistance sur ce point se veut un écho des luttes menées par les artistes professionnel-le's, notamment au sein d'une association comme THEMAA, pour la reconnaissance de leurs métiers, de leurs écritures, et surtout de leurs besoins spécifiques en termes d'espace, de temps et de moyens.

L'évolution de la condition matérielle de la figure marionnettique - passée d'objet sculpté à manifestation ondulatoire-renouvelle les besoins pratiques en termes d'accès à des espaces de répétitions équipés et de dépenses énergétiques, par exemple. En ce sens, l'écriture de l'infiguré n'évacue pas la question matérielle mais la pose différemment. Aussi une spécificité persiste, qui doit être reconnue pour permettre le développement et le soutien de ces écritures. La figure ondulatoire, sculptée par le son et la lumière, exige des espaces, des outils et des organisations singulières du travail autant de besoins liés de près à la place du geste plastique persistant au cœur des écritures marionnettiques.

Malgré la défiguration à l'œuvre dans les créations de Benjamin Verdonck, l'artiste témoigne par exemple du fait que des données matérielles ont été déterminantes dans l'écriture de Chansonnette pour Gigi. Accompagné dès l'origine par le créateur lumières, l'artiste a mené un long travail de recherche plastique en atelier. Tous deux y ont fait de nombreux essais non concluants car la qualité du matériau qu'ils avaient choisi pour construire le castelet mobile ne permettait pas des mouvements assez fluides des portes de bois : 
J'avais choisi du carton Honeycomb, qui est le matériau utilisé pour faire des portes. C'est donc assez rigide. [...] Le problème est que ce matériau est très sensible aux températures et comme ce sont des panneaux assez grands, mesurant un mètre sur un mètre vingt, ils avaient tendance à s'incurver. Comme ils doivent bouger dans des rails d'aluminium, la moindre torsion les fait bloquer. ${ }^{49}$ et du facteur de marionnettes: des données esthétiques et cinétiques doivent être prises en compte dans la construction. Il cherche précisément pour Chansonnette pour Gigi des mouvements assez fluides, des formes assez épurées et des couleurs assez neutres pour que ces objets puissent justement disparaitre à la vue ${ }^{50}$ et jouer ainsi au mieux leur rôle de seuils pour le regard.

La problématique plastique et son influence sur l'écriture dramatique apparaît autrement dans le contexte des tournées. À la question que nous lui posons sur ce qui a déterminé la taille du castelet de Chansonnette pour Gigi, Benjamin Verdonck répond que le critère de visibilité a dû s'adapter à celui de la transportabilité de l'objet:

Pour [...] le castelet, on a d'abord calculé ce qui pouvait entrer en largeur dans une camionnette normale et le poids qu'on pouvait y mettre. À partir de ça, on a pris en compte les questions de visibilité. On a pris les mesures de chaque salle et on a construit cet objet de manière à avoir la meilleure visibilité, tout en respectant la contrainte de la camionnette. ${ }^{51}$

Le processus de création de Chansonnette pour Gigi illustre ainsi le fait que l'évolution esthétique vers une figure davantage immatérielle n'évacue pas les contraintes pratiques posées par les objets du spectacle.

Olivier Vallet nous indique également à quel point l'évanescence de la figure est déterminée par une extrême rigueur technique, qui implique un travail permanent de réglage et d'adaptation pour le jeu, des machines de projection qu'il construit. Son rôle de constructeur des objets de Rêves et motifs n'est pas limité à une période qui précèderait l'écriture du spectacle. Olivier Vallet est présent en permanence au cours des répétitions. Ainsi peut-il adapter les mécanismes de ses constructions, les contrôles de ses machines, les tailles et la nature des écrans au jeu des interprètes. Son travail vise à mieux connaître la matière et les objets sans lesquels ne pourrait émerger une figure évanescente et fugace, qui parvient à hanter l'ensemble du plateau.

Affirmer le devenir immatériel de la figure marionnettique impose donc de ne pas négliger, en contrepoint, l'importance des conditions matérielles de production des œuvres. Des travaux sont en cours, notamment au sein de la chaire ICiMa, pour penser en termes systémiques l'éco-conception de marionnettes. Nous avons souligné en participant à ces chantiers de recherche la nécessité d'y prendre en compte les évolutions matérielles des écritures marionnettiques, à la fois pour que ces études fournissent des outils aux artistes qui explorent les territoires en marge de l'objetmarionnette, mais aussi pour ne pas laisser penser que l'évacuation de l'objet manipulé pourrait signifier l'évacuation des problématiques de santé et d'écologie liées aux arts de la marionnette ${ }^{52}$.

\section{Conclusion}

Afin d'appréhender les évolutions que connaissent les scènes contemporaines, le discours analytique sur les arts de la marionnette gagne à opérer ce décentrement par rapport à la notion d'objet-marionnette. Ce concept associant une fonction dramatique 
à un objet fixe mène à une impasse dans l'analyse de créations qui défont l'assise matérielle de la figure et jouent de sa circulation à travers les corps, les objets et les espaces. François Lazaro comme Les Rémouleurs mettent en drame la défection de la forme visible au profit d'une figure spatialisée, qui hante l'ensemble du dispositif. Gisèle Vienne, lorsqu'elle met en scène des mannequins anthropomorphes, défait ponctuellement la possibilité de projection d'une présence en eux. L'écriture de leur manipulation relève davantage d'un geste d'opacification, qui souligne leur qualité d'objets inertes. C'est enfin dans les espaces vides, entre les formes, que Benjamin Verdonck concentre le mouvement du drame. Son travail plastique vise à indiquer au regard spectatoriel un chemin vers l'infiguré et l'informe, ce qui ne se dit pas mais doit s'entendre à travers sa "chansonnette", à l'image de la violence des scènes implicitement rapportée à travers le regard de l'enfant dans le texte du spectacle.

Entrer dans ces créations par la question du statut de la matière nous rend sensible à une forme de résistance à la métaphorisation des objets. Ce détournement du fonctionnement iconologique des objets a une double conséquence. D'une part, la figure n'est pas matérialisée - sa présence acquiert une forme d'indépendance par rapport à toute assise matérielle. D'autre part, la matière fonctionne non plus comme « image de » mais acquiert un rôle dramaturgique lié à ses propriétés physiques et son irréductible être-là.

La matière qui «EST » en scène devient actrice d'un drame de la persistance, de la résistance contre l'effacement et la dispersion. Les écritures marionnettiques traversent ainsi de façon essentielle la question du vide scénique et de l'évanescence du geste dramatique, question qui traverse l'histoire des arts théâtraux. Nous touchons ainsi, avec cette tension vers l'évidement, à une forme très essentielle du drame tel qu'en parle Peter Brook dans L'Espace vide ${ }^{53}$ ou encore Denis Guénoun dans une perspective philosophique :

$\mathrm{Au}$ théâtre, ce qui vient sur scène est radicalement provisoire. Ce qui occupe le vide y sera vite évacué. Et le théâtre s'occupe à refaire le vide, sans cesse. Fini le jeu, on démonte. Place aux suivants. À nouveau le dégagement, le vidage. Le théâtre n'admet pas de comblement durable, stabilisé. [...] son vide doit être produit. Non pas un vide trouvé, disponible, dont on se contenterait de faire usage : mais un vide fait, travaillé, engendré par la régie de scène, parce qu'il n'est pas utilisé une fois, mais sans cesse re-produit, évacué à nouveau pour que la scène à nouveau s'emplisse d'actions et d'événements attendus. ${ }^{54}$

37 Avec lui, nous affirmons que la spécificité des créations marionnettiques contemporaines serait non pas d'élaborer des présences à partir des matières inertes mais bien d'animer ces matières pour mettre en scène, à partir d'elles et à vue, une production du vide. Le statut dramaturgique de la matière marionnettique passe ainsi de corps du sujet fictif à celui d'indice sensible d'une figuration en cours et d'un apparaître retenu, difficile, empêché, de la figure fictive. 


\section{NOTES}

1. Clastic Théâtre, dossier de présentation de la création Des hurlements montaient le long des saules pleureurs, 2013, p. 4.

2. Dans la suite de cet article, le titre de cette création sera abrégé sous la forme : les Hurlements.

3. Un cadre de bois soutient en son centre une structure circulaire et plane faite de fils et où est entretenu un film très fin de liquide savonneux. Pour le mettre au point, Olivier Vallet qui codirige la compagnie a collaboré avec François Graner, chercheur au CNRS et spécialiste des bulles de savon. Il a participé à l'amélioration d'un dispositif utilisé habituellement par les scientifiques pour étudier notamment les phénomènes météorologiques. L'innovation d'Olivier Vallet tient à l'alimentation en continu du film de savon, qui lui confère plus de résistance et de longévité.

4. Une telle définition de la marionnette comme objet rejoint celle donnée par Henryk Jurkowski en introduction de son ouvrage Métamorphoses : la marionnette au XXe siècle. La marionnette, écritil, est « une figure artificielle, articulée, fabriquée selon les principes des arts plastiques, dotée des capacités techniques pour être mise en jeu, lors d'un spectacle, devant un public, en tant que sujet fictif » (Métamorphoses : la marionnette au XXe siècle, Montpellier, L'Entretemps, 2008, p. 10).

5. Ce qui est évoqué ici comme une conception iconologique rejoint le principe des « deux corps de la marionnette ", exposé par Amos Fergombé dans le cadre du colloque «Corps vivants / corps marionnettiques ", organisé par Françoise Heulot-Petit et Stanka Pavlova, Université d'Artois, Arras, mars 2010. Ce principe est transposé des travaux de l'historien Ernst Kantor, qui dans son essai sur "les Deux Corps du roi», analyse les implications du principe médiéval juridicoreligieux de la double nature du roi (E. H. Kantorowicz, Les Deux Corps du roi : essai sur la théologie politique au Moyen âge, J.-P. Genet et N. Genêt (trad.), Paris, Gallimard, 1989).

6. F. de Mèredieu, Histoire matérielle et immatérielle de l'art moderne, Paris, Larousse, 2004, p. 13.

7. La médiologie, notamment fondée par Régis Debray, pose une distinction théorique entre les concepts de «média» et «médium». L'enjeu en est de ne pas réduire l'ensemble des «médiums» à un rôle communicationnel propre aux «médias ». C'est suivant ce principe que nous emploierons également le pluriel «médiums " plutôt que celui de "media " Les médiums désignent l'ensemble des moyens matériels par lesquels les images "prennent corps", autrement dit par lesquels elles acquièrent une visibilité et une matérialité (H. Belting, Pour une anthropologie des images, J. B. Torrent (trad.), Paris, Gallimard, 2004, p. 8).

8. Nous empruntons les termes $d$ ' «opacité » et de "transparence» au vocabulaire de la médiologie. Ils nous permettent de distinguer le médium qui permet l'élaboration-ou « animation » - des images par le regard et celui qui fait écran à une telle animation. Sur ce sujet, voir notamment R. Debray, Vie et mort de l'image. Une histoire du regard en Occident, Paris, Gallimard, 1992 ; et H. Belting, Pour une anthropologie des images, op. cit.

9. Le plasticien avait déjà collaboré avec le Clastic Théâtre sur de précédentes créations, telles Le Rêve de votre vie (1999) et Entre chien et loup $(1994$; 1997), mais il avait alors spécialement créé des objets articulés et destinés à être manipulés par des interprètes. Avec les Hurlements, François Lazaro met en scène des sculptures, des tableaux et des textes de Francis Marshall préexistant à son propre projet dramatique.

10. Nouzonville fait partie de ces communes ardennaises de la vallée de la Meuse dont l'économie était fondée au siècle dernier sur le travail du fer, et qui sont aujourd'hui fortement marquées par le chômage et l'appauvrissement économique de leurs habitant·e·s.

11. Clastic Théâtre, dossier de présentation de la création Des hurlements montaient le long des saules pleureurs, op.cit., p. 4. Remarquons que ce même terme de "bourrage" se rencontre également dans la pièce de Pauline Picot, Les Possibles de son corps. L'éclatement du corps de la poupée fonctionne dans ce monologue comme menace et comme fait de langue. Il agit en 
puissance et, retenu, il suscite une tension dramatique autour de l'évidement du corps : «Et le bourrage qui voudrait sortir par les yeux et les coutures qui voudraient craquer et tout ce qui voudrait sourdre de la peau en tordant les mailles, tout ce qui voudrait pouvoir se dire, quoi mais le bourrage reste bien en place et au contraire se comprime et se vide, et le vertige de ce vide se fait sentir dans une oscillation faible des fils » (P. Picot, Les Possibles de son corps, Fontenaysous-Bois, Quartett, 2012, p. 41).

12. En 1922, Oskar Schlemmer propose avec le Ballet triadique une danse des formes sur fonds de couleurs unies. Membre de l'école du Bauhaus, l'artiste allemand cherche à extraire la forme humaine de l'art chorégraphique et vise en cela un art du pur mouvement, dégagé des contraintes du corps. Les costumes portés par les danseur·se·s, créés par Oskar Schlemmer luimême, sont des œuvres plastiques tridimensionnelles, qui effacent au moins en partie les silhouettes des interprètes au profit de volumes géométriques. L'écriture chorégraphique repose ainsi sur les relations et distances entre les formes, sur leurs dynamiques propres, leurs trajectoires et le rythme également induit par leurs couleurs.

13. B. Verdonck, entretien réalisé par Julie Postel, 2018. Transcription publiée en annexe de : J. Postel, Présences de la marionnette contemporaine : figure, figuration, défiguration, thèse de doctorat en Arts du spectacle, Valenciennes, Université Polytechnique Hauts-de-France, 2019.

14. Id.

15. Id.

16. Émilie Valantin pour la première fois avait mis en scène des marionnettes de glace pour monter Le Cid de Pierre Corneille, en 1996.

17. Voir à ce sujet la présentation du cycle I.C.E. sur le site de la compagnie Non Nova. URL : <http://www.cienonova.com/i/i-c-e/>. Consulté le 13 février 2019.

18. V. Novarina, Le Théâtre des oreilles - pièce radiophonique pour marionnette électronique, Charleville-Mézières, Institut International de la Marionnette, 2001, p. 11.

19. A. Grothendieck, Récoltes et Semailles. Réflexions et témoignage sur un passé de mathématicien, texte inédit, 1985. Disponible en ligne. URL: <http://archive.wikiwix.com/cache/? url=https\%3A\%2F\%2Fjmrlivres.files.wordpress.com\%2F2009\%2F11\%2Frecoltes-et-semailles.pdf>. Consulté le 14 février 2019.

20. Précisons que dans le sens où nous l'employons, l'expression "devenir-image » de l'objetmarionnette ne désigne pas un processus historique. Cela reviendrait à oublier la longue tradition des très variés théâtres d'ombres existant à travers le monde. Le « devenir-image » que nous pointons est davantage un principe esthétique, marqué par la tension entre l'objet et son image et par la mise en drame de l'incertaine densité matérielle des figures. Ce « devenir » se joue en fait au sein même de la dramaturgie d'une œuvre pour évoquer un monde moins tangible, qui échappe à la réalité matérielle la plus immédiate.

21. G. Vienne, entretien réalisé par Julie Postel, 2018.

22. Id.

23. Id.

24. C. Grazioli, «Souffles de lumière : animer les choses », L. Van Goethem (trad.), dans S. MartinLahmani (éd.), Poétiques de l'illusion. Dialogues contemporains entre marionnette et magie, Bruxelles, Alternatives théâtrales, 2018, p. 87.

25. Id.

26. Id.

27. Id.

28. C. Grazioli, "Paysages avec marionnettes: Rainer Maria Rilke et la scène", dans H. Beauchamp et al. (dir.), Les Scènes philosophiques de la marionnette, Montpellier: L'Entretemps, Charleville-Mézières : Institut International de la Marionnette, 2016, p. 111.

29. M. Maeterlinck, «Menus propos : le théâtre », La Jeune Belgique, septembre 1890, p. 335. Cité par C. Grazioli, « Cavités lumineuses, reflets d'ombres : présences absentes et dramaturgies de la 
lumière ", in C. Beaufort et $\mathrm{M}$. Lebrère (dir.), Ambivalences de la lumière, Pau, Presses de l'Université de Pau et des Pays de l'Adour, 2016, p. 169.

30. Ces illusions optiques gardent une dimension artisanale qui permet à tout moment aux spectateur-trice's de les mettre à distance s'ileelle-s le souhaitent. Benjamin Verdonck est en effet visible sur le côté du castelet lorsqu'il manipule les plans de bois grâce à des ficelles. On pourrait parler à cet endroit d'une illusion consentie.

31. C. Grazioli, « Paysages avec marionnettes : Rainer Maria Rilke et la scène », op. cit., p. 111.

32. V. Novarina, Le Théâtre des oreilles - pièce radiophonique pour marionnette électronique, op. cit., p. 11.

33. A. Artaud, Le Théâtre et son double. Suivi de : le théâtre de Séraphin, Paris, Gallimard, 1981, p. 136.

34. Quoiqu'absente des créations d'Antonin Artaud, la marionnette imprègne, comme principe et à travers l'image du pantin, sa conception philosophique du drame. Sur ce sujet, voir notamment l'article d'Hélène Beauchamp, «Envers et renversements de la marionnette chez Antonin Artaud : la fin d'un mythe de la modernité », Théâtre/Public, nº 193, 2009, pp. 7-11.

35. Ibid., p. 102-103. (Nous soulignons.)

36. F. de Mèredieu, Arts et nouvelles technologies: art vidéo, art numérique, Paris, Larousse, 2003, p. 164.

37. Ibid., p. 217-218.

38. Des ingénieurs de l'Institut de Recherche et Coordination Acoustique/Musique (Ircam) ont utilisé pour la création sonore de The Pyre de Gisèle Vienne un système de diffusion du son, appelé «Spat» (pour "spatialisation»), qui «permet de sculpter la musique dans l'espace en faisant surgir le son à des endroits précis. [...] Comme si un instrument fantôme apparaissait tout d'un coup dans tel ou tel endroit du plateau » (extrait d'entretien avec Gisèle Vienne, réalisé par Julie Postel, 2018).

39. O. Vallet, entretien réalisé par Julie Postel, 2017. Transcription publiée en annexe de: J. Postel, Présences de la marionnette contemporaine : figure, figuration, défiguration, thèse de doctorat en Arts du spectacle, Valenciennes, Université Polytechnique Hauts-de-France, 2019.

40. Voir également le film réalisé par P. Meunier (compagnie La Belle Meunière), Ça continue!, Besançon, Les Solitaires Intempestifs, 2008, 110 min. Extrait visible en ligne, le mouvement « Ça pousse ! ». URL : <https://www.youtube.com/watch?v=yvO1jqrpoRE>. Consulté le 15 février 2019.

41. G. Vienne, "Showroomdummies », sur le site Gisèle Vienne. URL : <http://www.g-v.fr/fr/ shows/showroomdummies-2/>, s. d. Consulté le 11 février 2019.

42. R. Castelli et al., catalogue de l'exposition Robotic art = Art robotique présentée à la Cité des Sciences et de l'Industrie à Paris du 8 avril 2014 au 4 janvier 2015, Paris, Art Book Magazine et Cité des Sciences et de l'Industrie, 2014, p. 62.

43. J.-L. Mattéoli, L'Objet pauvre: mémoire et quotidien sur les scènes contemporaines françaises, Rennes, Presses universitaires de Rennes, 2011, p. 243.

44. Id.

45. M.-J. Mondzain, Le Commerce des regards, Paris, Seuil, 2003, p. 39.

46. T. Kantor, Le Théâtre de la mort, D. Bablet (dir.), Lausanne, Suisse, L'Âge d'homme, 2004, p. 167.

47. M.-J. Mondzain, Le Commerce des regards, Paris, Seuil, 2003, p. 17.

48. Id..

49. B. Verdonck, op. cit.

50. Bien qu'ils restent visibles. Nous jouons ici sur la distinction, notamment éclairée par MarieJosé Mondzain entre le visible et la vision, entre ce qui est visible et ce qui est vu.

51. Id.

52. Sur ce point, voir notamment les articles « Journal de bord » du chantier sur l'éco-conception de marionnettes (porté par la chaire ICiMa), en ligne sur le blog consacré à ce dispositif de recherche. URL : <https://icima.hypotheses.org/?s=journal+de+bord+eco-conception>, consulté le 27 mai 2019. 
53. P. Brook, L'Espace vide. Écrits sur le théâtre, C. Estienne et F. Fayolle (trad.), Paris, Seuil, 1977.

54. D. Guénoun, "Qu'est-ce qu'une scène? ", dans Collectif, Philosophie de la scène, Besançon, Les Solitaires intempestifs, 2010, p. 17.

\section{RÉSUMÉS}

Des créations contemporaines aussi variées esthétiquement que celles de Gisèle Vienne, de François Lazaro (Clastic Théâtre), de Benjamin Verdonck ou de la compagnie Les Rémouleurs nous révèlent une révolution en cours dans les arts de la marionnette : l'objet mis en scène n'y est plus le lieu de projection d'un sujet fictif. Si ces écritures continuent de croiser un travail plastique au geste dramatique, l'objet n'y fait pas corps; des présences se font davantage sentir entre les corps et les objets, par le mouvement des choses, la suggestion d'un vivant invisible. Cette défection de la marionnette comme objet a deux conséquences esthétiques. D'une part, la figure marionnettique adopte un caractère ondulatoire voire immatériel. D'autre part, la matière, qui n'est plus transfigurée, persiste comme relai nécessaire à la perception d'une présence. Elle agit alors en négatif, par son opacité et sa résistance à l'animation. Les « écritures de l'infiguré » reposent ainsi sur un nécessaire "passage» par la matière, reformulant esthétiquement, techniquement et politiquement les contraintes matérielles liées à la création marionnettique.

\section{INDEX}

Mots-clés : marionnette contemporaine, figure, dramaturgie, ondulatoire, présence, conditions matérielles 